\title{
Avoided deconfinement in Randall-Sundrum models
}

\author{
Prateek Agrawal and Michael Nee \\ Rudolf Peierls Centre for Theoretical Physics, University of Oxford, \\ Parks Road, Oxford OX1 3PU, U.K. \\ E-mail: prateek.agrawal@physics.ox.ac.uk, \\ michael.nee@physics.ox.ac.uk
}

ABSTRACT: We study first order phase transitions in Randall-Sundrum models in the early universe dual to confinement in large- $N$ gauge theories. The transition rate to the confined phase is suppressed by a factor $\exp \left(-N^{2}\right)$, and may not complete for $N \gg 1$, instead leading to an eternally inflating phase. To avoid this fate, the resulting constraint on $N$ makes the RS effective field theory only marginally under control. We present a mechanism where the IR brane remains stabilized at very high temperature, so that the theory stays in the confined phase at all times after inflation and reheating. We call this mechanism avoided deconfinement. The mechanism involves adding new scalar fields on the IR brane which provide a stablilizing contribution to the radion potential at finite temperature, in a spirit similar to Weinberg's symmetry non-restoration mechanism. Avoided deconfinement allows for a viable cosmology for theories with parametrically large $N$. Early universe cosmological phenomena such as WIMP freeze-out, axion abundance, baryogenesis, phase transitions, and gravitational wave signatures are qualitatively modified.

Keywords: Confinement, Cosmology of Theories beyond the SM

ArXiv EPRINT: 2103.05646 


\section{Contents}

1 Introduction 1

2 The supercooled Randall-Sundrum model $\quad 4$

2.1 (De)confinement phase transition in the RS model 6

$\begin{array}{lll}2.2 & \text { Bounce action from the radion } & 7\end{array}$

3 5D model for avoided deconfinement $\quad 10$

3.1 Finite temperature effective potential for the radion 12

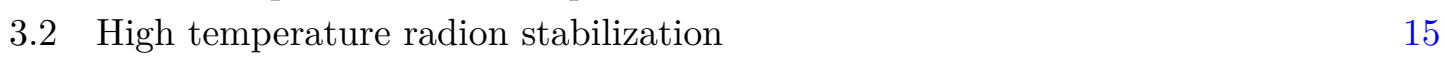

4 Low temperature phenomenology 17

$\begin{array}{ll}4.1 \text { Effective Lagrangian at zero temperature } & 17\end{array}$

$\begin{array}{lll}4.2 & \text { Experimental constraints } & 18\end{array}$

5 Cosmology 19

5.1 Electroweak phase transition 20

5.2 High scale baryogenesis $\quad 21$

5.3 WIMP freeze-in 22

5.4 QCD phase transition and the QCD axion 23

5.5 Gravitational waves 24

6 Discussion $\quad 24$

$\begin{array}{ll}\text { A Bounce action for deconfining phase transition } & 25\end{array}$

\section{Introduction}

Large- $N$ gauge theories are important both theoretically as well as phenomenologically. The large- $N$ limit makes many problems in strongly-coupled gauge theories tractable, providing an expansion parameter for non-Abelian theories [1]. It remarkably also plays a central role in gauge-gravity duality $[2-4]$ where a large- $N$ gauge theory is dual to a gravitational theory in higher dimensions. A major phenomenological application of the duality appears in the context of the Randall-Sundrum (RS) model [5]. The RS model provides an elegant solution to the hierarchy problem, with an exponential separation of the Planck and weak scales that is generated by the warping of the extra dimension. The ratio of the two scales is set by the size of the extra dimension, which is fixed by introducing a stabilisation mechanism, generating a potential for the radion [6]. This mechanism is dual to adding an almost-marginal operator in the gauge theory which explicitly breaks the scale invariance of the theory. The small anomalous dimension for this operator, equivalent to 
a small bulk mass for the stabilising field [3], generates an exponentially small scale in the IR in a manner analogous to dimensional transmutation in QCD.

The RS model also provides an effective description for a number of string constructions of realistic vacua with reduced supersymmetry [7-11]. In the large- $N$ limit, these explicit constructions are described by an effective quantum field theory where gravity is weakly coupled. A prominent example is the KKLT scenario [12], which is partly based on the Klebanov-Witten [13] and Klebanov-Strassler [14, 15] constructions. For this construction the stability of the de Sitter vacuum is often justified in the probe approximation which is valid for parametrically large $N[16,17]$. Therefore, large- $N$ gauge theories with gravitational duals are expected to be an important part of a UV complete description of our universe.

Theories which are described by the RS model in an appropriate limit suffer from a severe cosmological constraint. At high temperature the gauge theory is in the deconfined phase, and the confined phase becomes thermodynamically preferred below a critical temperature. However, the confinement phase transition is first order and exponentially suppressed with a rate proportional to $\exp \left(-N^{2} / \lambda\right)$, where $\lambda$ denotes a possible weak coupling. The gravitational description of the deconfined phase is the AdS-Schwarzschild (AdS-S) solution with a UV brane. The confinement transition corresponds to the appearance of the IR brane from behind the AdS-S horizon [18-20]. The confinement scale in the gauge theory is dual to the vacuum expectation value of the radion field which sets the size of the extra dimension in the RS model.

For large- $N$, the suppressed phase transition is much slower than the expansion rate of the universe, leading to eternal inflation. Requiring the phase transition to complete leads to a robust upper bound on $N[21]$ :

$$
N \lesssim \sqrt{4 \lambda \log \frac{M_{\mathrm{pl}}}{\Lambda_{c}}} \sim 12 \sqrt{\lambda}
$$

where $\Lambda_{c} \simeq 1 \mathrm{TeV}$ is the confinement scale for the gauge theory. This bound follows just from dimensional analysis and is independent of the details of the RS model. The ratio $N / 4 \pi$ sets the hierarchy between the curvature scale $k$ and the $5 \mathrm{~d}$ reduced Planck scale $M_{5}, N / 4 \pi \sim\left(M_{5} / k\right)^{3 / 2}[22]$. A small value of this ratio, as implied by the bound (1.1), then means that corrections due to Planck scale physics become important, making the EFT control in the RS model delicate. Gravitational loop corrections can be estimated by the following loop counting parameter,

$$
\frac{N_{\text {species }} k^{3}}{16 \pi^{2} M_{5}^{3}}<1 \Rightarrow N^{2} \gtrsim N_{\text {species }}
$$

which is in tension with equation (1.1) even with just the SM degrees of freedom contributing to $N_{\text {species }} \sim 100$.

In fact, the bound is much more stringent within the simplest version of the RS model. In this setup, the backreaction of the stabilization mechanism and breaking of scale invariance are assumed to be small even close to the confinement scale. The gauge theory is an 
approximately conformal field theory $(\mathrm{CFT})$, with spontaneously broken conformal invariance in the confined phase. The approximate conformality suppresses the phase transition further, making $\lambda \ll 1$, so that the bound on $N$ in equation (1.1) is impossible to satisfy.

There is a large body of work devoted to relaxing this more stringent constraint on the RS model by changing the details of the stabilisation mechanism [23-38] in such a way that $\lambda \simeq 1$ and the phase transition occurs more rapidly. However, the $N^{2}$ dependence of the tunnelling rate is a generic feature of the confinement phase transition. While modifying the stabilisation mechanism can change the numerical value of the bound on $N$, in all these models the phase transition is exponentially suppressed at large- $N$ and therefore subject to the bound in equation (1.1). As pointed out in [39], for Klebanov-Strassler type constructions, the effective value of $N$ itself varies over the extra-dimensional coordinate - the relevant $N$ in this case is the value near the confinement scale.

In this paper we present a simple modification to the RS model where $N$ can be made parametrically large without running into this cosmological bound. We construct a scenario where the confinement scale grows with temperature, and hence the universe can remain in the confined phase at all times in early cosmology. For this reason, we call our mechanism avoided deconfinement. In order to achieve this we consider the RS I model with the IR brane stabilised by a Goldberger-Wise (GW) field $\Phi[6]$. By introducing new scalars to the IR brane, we can generate a potential which stabilises the IR brane at high temperatures. The mechanism we use to achieve this is reminiscent of non-restoration of electroweak symmetry at high temperatures, as considered in refs. [40-44]. Similar mechanisms have also been proposed to avoid monopoles [45-47] or domain walls [48] in Grand Unified theories, as models of $\mathrm{CP}$ violation at high temperature [49,50], and in the $O(N) \times O(N)$ models of $[51,52]$.

The modification we make to the RS model can lead to dramatic departures from its standard cosmological history. Above a critical temperature $T_{c}$, the confinement scale varies almost linearly with temperature $T$ leading to $T$-dependent mass scales on the IR brane,

$$
M_{\mathrm{ir}}(T) \propto \mu(T)=\mu(0)\left(\frac{T}{c T_{c}}\right)^{1 /(1+\epsilon)}
$$

with $\epsilon \ll 1, c \sim \mathcal{O}(1)$. For a mass scale $M_{\text {ir }}>T_{c}$, this can imply that the ratio $T / M_{\text {ir }}(T)$ reaches 1 at very high temperatures, or potentially not at all (similar to low reheating temperature models). Taking the standard model (SM) to be localised on the IR brane, the $T$-dependence of the electroweak and QCD scales is as in equation (1.3). If $v_{\mathrm{ew}}>T_{c}$, the electroweak phase transition occurs at temperatures far above the $\mathrm{TeV}$ scale or is completely avoided. Furthermore, at high temperature, fields localised in the UV of the RS model may have had significant overlap with fields localised towards the IR of the theory, a feature which may have applications to models of baryogenesis and dark matter production.

The initial condition for our mechanism to work is that the universe exits inflation in the RS phase with a stabilized IR brane. A relatively simple way to achieve this is to have inflation with Hubble rate below the confinement scale of the gauge group, or with an additional stabilization of the IR brane during inflation. It will be an interesting future direction to study the interplay of $\mathrm{AD}$ with inflationary models. After inflation the universe 

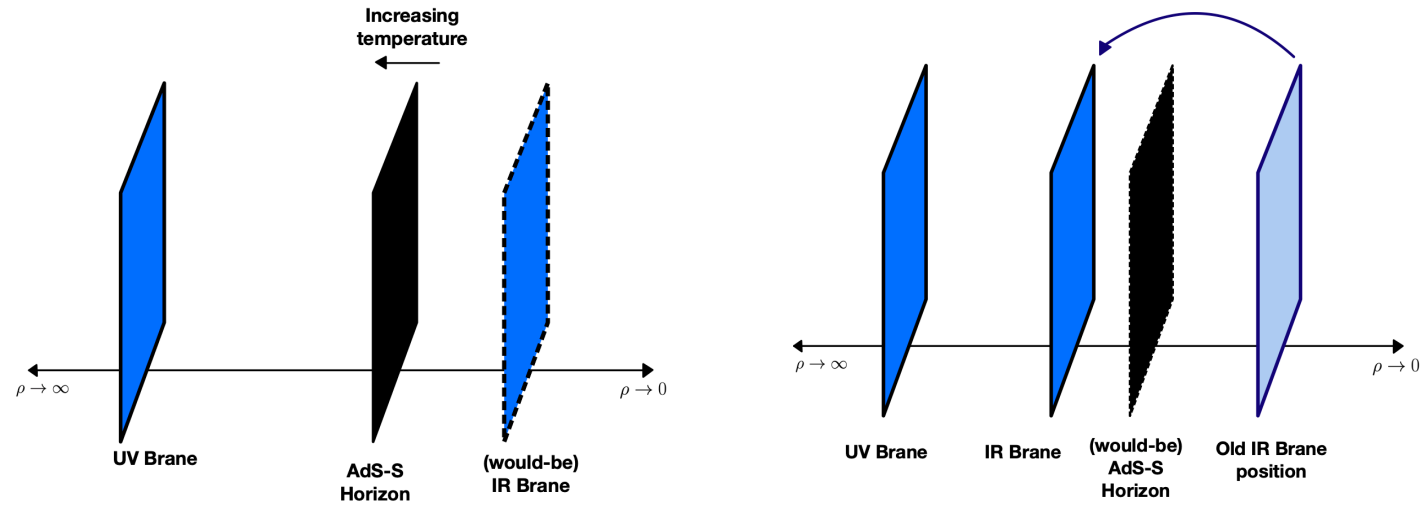

Figure 1. Left hand diagram shows the high temperature behaviour of the RS model, while the right hand diagram shows the high temperature behaviour in the $\mathrm{AD}$ model. Beyond a certain temperature in the RS model the IR brane is stabilised behind the location of the horizon in the AdS-S phase, indicating that the model is unstable against black hole formation. In the AD model, this instability is lifted by introducing a temperature dependence to the stabilisation mechanism in such a way that the IR brane is stabilised outside the would-be horizon at high temperatures.

reheats and the $\mathrm{AD}$ mechanism prevents the brane from falling behind the would-be AdS-S horizon (see figure 1). Note that at high enough temperatures the AdS-S phase will still be the preferred thermodynamic phase of the theory, but in the avoided deconfinement model the RS phase is classically stable. The probability of tunnelling from the RS to the AdS-S phase is exponentially suppressed by $N^{2}$ factors, and can be made vanishingly small in the large- $N$ limit.

The rest of this paper is organised as follows. In section 2 we describe the early universe cosmology and summarise the details of the confinement phase transition in various generalizations of the RS model that have been considered in the literature. We go on to describe the avoided deconfinement (AD) model in section 3 and show how the model leads to a stabilised IR brane at high temperatures. In section 4 we present the low energy effective Lagrangian and discuss some of the experimental signatures of the model. In section 5 we then discuss the unique early universe cosmology of the model and how this relates to other non-standard cosmological histories in the literature. We also discuss potential applications of the model to baryogenesis, dark matter production and potential gravitational wave signatures in this section, before concluding and summarising in section 6 .

\section{The supercooled Randall-Sundrum model}

In this section we review the standard cosmology of the RS type I model and GoldbergerWise field, and its dual gauge theory description via the gauge-gravity duality. In the standard treatment of gauge-gravity duality at finite temperature, the gauge theory partition function is defined on a manifold $\mathcal{M}=S_{1} \times R^{3}$ with the temporal direction compactified on a circle of radius $\beta=\pi / T$. The corresponding gravitational theory is defined on a 5-dimensional manifold with $\mathcal{M}$ as the boundary. In computing the gravitational partition function, all possible geometries $\Sigma$ which satisfy the boundary condition $\partial \Sigma=\mathcal{M}$ must be 
integrated over [19]. The partition function will however be dominated by classical gravity solutions. Each semi-classical gravitational solution $\Sigma_{i}$ which satisfies the boundary condition is interpreted as a different phase of the CFT. At a given temperature, the geometry which minimises the Euclidean action will give the dominant contribution to the partition function, and therefore correspond to the preferred phase of the CFT.

In the RS model, the UV brane cuts off the AdS space, and hence plays the role of the boundary $\partial \Sigma$. The dual gauge theory is interpreted as a field theory coupled to $4 \mathrm{D}$ gravity, defined on the manifold $\mathcal{M}$. One of the possible classical solutions is,

$$
d s_{\mathrm{RS}}^{2}=k^{2} \rho^{2} d t^{2}-\frac{d \rho^{2}}{k^{2} \rho^{2}}-\rho^{2} k^{2} d x_{i}^{2}
$$

with the space in the $\rho$ direction cut off at the position of the IR and UV branes so that $\rho_{\text {ir }}<\rho<\rho_{\text {uv }}$. Here and throughout this paper, we work in a frame where $\rho_{\mathrm{uv}}$ is fixed to the value $\rho_{\mathrm{uv}}=k^{-1}$, where $k$ is the AdS curvature. A convenient definition of the temperature of the $5 \mathrm{D}$ theory is the local temperature at the UV brane. We will simply refer to this temperature as $T$. Thermal effects tend to push the IR brane towards the horizon, rendering the RS solution unstable [20] at arbitrarily small temperatures in the absence of stabilization. This instability can be lifted using the GW mechanism [6].

The quasi-conformal theory dual to the RS model is a strongly coupled gauge theory $[53,54]$ with $\mathcal{O}\left(N^{2}\right)$ degrees of freedom, where $N$ can be determined by matching the entropy of the black hole with the entropy of the high temperature phase of the gauge theory [22],

$$
\frac{N^{2}}{16 \pi^{2}} \simeq 12\left(\frac{M_{5}}{k}\right)^{3}
$$

This relation can be modified by $\mathcal{O}(1)$ factors depending on strong coupling effects in different gauge theory models. We see that the large- $N$ aspect of the $4 \mathrm{D}$ gauge theory is a crucial feature of these models, since it corresponds to the hierarchy between the curvature scale $k$ and the $5 \mathrm{D}$ Planck scale $M_{5}$ in the $5 \mathrm{D}$ gravitational theory. The $\rho$ direction can be thought of as the RG scale of the conformal theory, with small $\rho$ corresponding to the IR of the theory. The UV and IR branes of the RS model correspond to UV and IR cutoffs in the gauge theory. The cutoff at the IR brane represents a spontaneous breaking of conformality in the IR due to confinement in the gauge theory, while the UV brane represents explicit breaking by the cutoff at the Planck scale [22]. The RS model with the IR brane therefore corresponds to the confined phase of the conformal theory. The GW mechanism corresponds to introducing a nearly marginal operator to the CFT which explicitly breaks the conformal symmetry of the theory. The coupling of this operator is dual to a scalar field in the RS model with a small bulk mass. Introducing the GW scalar generates an effective potential for the radion (identified with $\mu=k^{2} \rho_{\text {ir }}$ in co-ordinates where the location of the UV brane is fixed [55]), with a minimum at small $\mu$ - the IR brane will then be stabilised at the minimum of this potential.

The RS solution with the IR brane becomes classically unstable at high temperatures. There is another classical solution that contributes to the finite temperature partition 
function given by the AdS-Schwarzschild (AdS-S) geometry,

$$
d s_{\text {AdS-S }}^{2}=f(\rho) d t^{2}-\frac{d \rho^{2}}{f(\rho)}-\rho^{2} k^{2} d x_{i}^{2}, \quad f(\rho)=k^{2}\left(\rho^{2}-\frac{\rho_{h}^{4}}{\rho^{2}}\right) .
$$

The position of the horizon $\rho_{h}$ is set by the temperature $\rho_{h}=\pi T / k^{2}$. The solution is cut off at $\rho=\rho_{\text {uv }}$ by the UV brane as before. The AdS-S solution is dual to the deconfined phase of the gauge theory, with the Hawking temperature and entropy of the AdS black hole equal to the corresponding quantities in the gauge theory. The AdS-S solution is classically stable for any non-zero temperature, and is the thermodynamically preferred phase of the theory at high temperatures.

As the universe cools below a critical temperature, the RS phase with the IR brane becomes preferred and there is a first order phase transition between the two phases which proceeds through a tunnelling process connecting the two solutions. This tunnelling process is strongly suppressed, however, due to the large change in free-energy in the two phases. The requirement that this phase transition completes places bounds on $N$ for the model to be cosmologically viable. These bounds typically require $N \sim \mathcal{O}(1)$, which is in tension with the assumption of working in the large- $N$ limit.

\section{1 (De)confinement phase transition in the RS model}

We consider the RS model with the GW stabilization mechanism. The bulk Lagrangian contains gravity and the GW field $(\Phi)$,

$$
S_{\text {bulk,RS }}\left[G_{A B}, \Phi\right]=\int d^{4} x d \rho \sqrt{G}\left[-2 M_{5}^{3} R+24 M_{5}^{3} k^{2}+\frac{1}{2} G^{A B} \partial_{A} \Phi \partial_{B} \Phi-\frac{1}{2} m_{\Phi}^{2} \Phi^{2}\right] .
$$

We also include brane localized terms,

$$
\begin{aligned}
S_{\mathrm{uv}, \mathrm{RS}} & =\int d^{4} x \sqrt{-g_{\mathrm{uv}}}\left[-\lambda_{\mathrm{uv}}\left(\Phi^{2}-v_{\mathrm{uv}}^{2}\right)^{2}-24 M_{5}^{3} k+\delta \Lambda_{\mathrm{uv}}\right] \\
S_{\mathrm{ir}, \mathrm{RS}} & =\int d^{4} x \sqrt{-g_{\mathrm{ir}}}\left[-\lambda_{\mathrm{ir}}\left(\Phi^{2}-v_{\mathrm{ir}}^{2}\right)^{2}+24 M_{5}^{3} k+\delta \Lambda_{\mathrm{ir}}\right] .
\end{aligned}
$$

where $g_{\mathrm{uv}, \text { ir }}$ are the induced metrics on the UV and IR branes. In the presence of the GW stabilization mechanism, only one combination of the brane tension detuning parameters $\delta \Lambda_{i}$ needs to be tuned, corresponding to the tuning of the $4 \mathrm{D}$ cosmological constant. Depending on the sign of $m_{\Phi}^{2}$, these parameters may be required to lie in a certain range for there to be a local minimum for the radion away from $\mu=0$. For simplicity, here we set each of the detuning parameters to 0 and assume $m_{\Phi}^{2}>0$. We also assume that the stabilization occurs in the limit of small backreaction. These assumptions are not crucial and do not affect the qualitative results. With these assumptions, the metrics in equations (2.1) and (2.3) continue to be approximate classical solutions.

We can obtain the potential for the radion by integrating over the classical solution for the GW field. In the limit where the brane localized terms fix $\Phi\left(\rho_{\mathrm{uv}, \text { ir }}\right)=v_{\mathrm{uv}, \text { ir }}$, the 4D effective potential for the radion, $\mu \equiv k^{2} \rho_{\text {ir }}$ is [20],

$$
V(\mu)=\epsilon v_{\mathrm{uv}}^{2} k^{4}+\left[(4+2 \epsilon) \mu^{4}\left(v_{\mathrm{ir}}-v_{\mathrm{uv}}(\mu / k)^{\epsilon}\right)^{2}-\epsilon v_{\mathrm{ir}}^{2} \mu^{4}\right]+\mathcal{O}\left(\mu^{8} / k^{4}\right),
$$


with $\epsilon=\sqrt{4+m_{\Phi}^{2} / k^{2}}-2$. The minimum is obtained for:

$$
\begin{aligned}
\mu_{\mathrm{TeV}} & =f(\epsilon) k\left(\frac{v_{\mathrm{ir}}}{v_{\mathrm{uv}}}\right)^{1 / \epsilon}, \\
f(\epsilon) & =\left[\frac{4+\epsilon+\sqrt{\epsilon(4+\epsilon)}}{4+2 \epsilon}\right]^{1 / \epsilon} \sim \mathcal{O}(1) .
\end{aligned}
$$

A relatively modest hierarchy in $v_{\mathrm{uv} \text {,ir }}$ and $\epsilon \sim 1 / 10$ can generate an exponential hierarchy between $k$ and $\mu_{\mathrm{TeV}}$. At energies $\lesssim \mu_{\mathrm{TeV}}$ the effective theory is a $4 \mathrm{D}$ theory with a tower of Kaluza-Klein $(\mathrm{KK})$ states with masses $\sim \mu_{\mathrm{TeV}}$. In the $4 \mathrm{D}$ theory, dimensionful parameters involving fields localized on the IR brane, such as the Higgs mass parameter, scale with $\mu_{\mathrm{TeV}}$, thus explaining the electroweak hierarchy elegantly.

At low-temperatures, $T<\mu_{\mathrm{TeV}}$, both classical solutions - the stabilized RS solution with UV and IR branes, and the AdS-S solution with a UV brane and a black hole horizon - are (meta)stable. However, at high-temperatures $T \gg \mu_{\mathrm{TeV}}$, the minimum of the radion potential is behind the AdS-S horizon $\mu_{\mathrm{TeV}}<\rho_{h} k^{2}$, indicating that the AdS-S solution is the only classical solution. During the early universe the universe is in the AdS-S phase; to get to the RS phase the universe needs to undergo a first order phase transition.

The tunnelling rate per unit volume for the phase transition is,

$$
\Gamma \simeq R_{c}^{-4} \exp \left(-S_{b}\right)
$$

where $S_{b}$ is the bounce action for the tunnelling transition, and $R_{c}$ is the radius of the critical bubble [56, 57]. The field configuration for the transition from the AdS-S phase to the RS phase involves moving the black hole horizon to the far IR, $\rho_{h} \rightarrow 0$, and then nucleating the IR brane at $\rho=0$ and bringing it to larger values of $\rho$. Therefore this field configuration probes the geometry in the region where the local temperature is superPlanckian and stringy corrections would be relevant. However, in the case where the transition temperature is low, and there is an approximate conformal symmetry in the IR, the dominant contribution to the bounce is dictated by the radion dynamics and can be estimated while ignoring the gravitational contribution to the bounce [38]. Even so, since the field configuration probes the geometry in the far IR, the bounce action for this configuration can depend sensitively on the details of the GW stabilisation, and other physics in the IR. We summarize the results for the bounce action that have been considered in various limits in the literature next.

\subsection{Bounce action from the radion}

In a large class of models, the phase transition is captured by the dynamics of the radion $[20,34,38]$. The general radion effective field theory can be understood in terms of the dual 4D theory. The 4D theory is a near-conformal field theory coupled to gravity. The gravitational sector breaks the conformal symmetry explicitly, but below the gravitational cutoff an approximate conformal symmetry survives. For a stabilised RS geometry with a light radion, the $4 \mathrm{D}$ effective theory below the $\mathrm{KK}$ scale $\mu_{\mathrm{TeV}}$ is well-described by an 
effective theory of a spontaneously broken (approximate) conformal symmetry, with the light radion/dilaton as the pseudo-Nambu-Goldstone boson.

In this section we work in this $4 \mathrm{~d}$ picture to study a few such generalisations that have been studied in the literature. As we will see, in each case the first order phase transition is highly suppressed. The effective Lagrangian for the dilaton $[20,58]$ can be written as,

$$
\mathcal{L}_{\text {eff }}=\frac{N^{2}}{16 \pi^{2}}\left[(\partial \mu)^{2}-\lambda(g(\mu)) \mu^{4}\right]
$$

where the $\mu$-dependence in $g(\mu)$ denotes the explicit breaking of conformal symmetry due to the GW deformation. We expect the dilaton to be the lightest bound state of the gauge theory $[59,60]$ as it is the pNGB of the broken dilation symmetry, so is the only relevant degree of freedom in the IR of the theory. The $N^{2}$ factor makes explicit the fact that the dilaton is interpreted as a glueball state in a $4 \mathrm{D}$ large- $\mathrm{N}$ gauge theory.

The free energy in the (de)confined phase is well approximated by,

$$
\begin{gathered}
F_{\text {confined }}=V\left(\mu_{\mathrm{TeV}}\right)=-\frac{N^{2}}{16 \pi^{2}} \lambda_{\mathrm{TeV}} \mu_{\mathrm{TeV}}^{4}+V_{0} \\
F_{\text {deconfined }}=C-2 \pi^{4}\left(M_{5} / k\right)^{3} T^{4}=C-\frac{\pi^{2}}{96} N^{2} T^{4}
\end{gathered}
$$

where we have defined $\lambda_{\mathrm{TeV}} \equiv\left|\lambda\left(g\left(\mu_{\mathrm{TeV}}\right)\right)\right|$, and added a constant $V_{0}$ to ensure that the vacuum energy at the minimum is zero. Notice that $\lambda\left(g\left(\mu_{\mathrm{TeV}}\right)\right)<0$ for $\mu_{\mathrm{TeV}}$ to be the minimum of the potential. $C$ can be calculated by matching the free energy at $\mu=T=0$. The critical temperature can be calculated by equating the free energy in the two phases at the transition,

$$
\begin{aligned}
C-\frac{\pi^{2}}{96} N^{2} T_{c}^{4} & \simeq-\frac{N^{2}}{16 \pi^{2}} \lambda_{\mathrm{TeV}} \mu_{\mathrm{TeV}}^{4}+V_{0} \\
\Rightarrow T_{c} & \simeq\left[\frac{6 \lambda_{\mathrm{TeV}}}{\pi^{4}}\right]^{1 / 4} \mu_{\mathrm{TeV}} .
\end{aligned}
$$

When $\lambda_{\mathrm{TeV}} \ll 1$, the transition temperature $T_{c} \ll \mu_{\mathrm{TeV}}$, and the approximation of radion domination is well justified.

If the phase transition is prompt, it completes for $T \sim T_{c}$. In this case the bubble has $\mathrm{O}(3)$ symmetry, and the action can be estimated in the thin-wall regime (see e.g. [21]),

$$
\frac{S_{3}}{T} \sim \frac{N^{2}}{8}\left[\frac{1}{\lambda_{\mathrm{TeV}}}\right]^{3 / 4} \frac{T_{c}}{T}\left(1-\left(\frac{T}{T_{c}}\right)^{4}\right)^{-2} .
$$

This explicitly shows the general enhancement of the bounce action by $N^{2}$, and often also by the weak coupling $\lambda_{\mathrm{TeV}}$.

We can evaluate the bounce action for the GW model considered in section 2.1 above. The quartic $\lambda_{\mathrm{TeV}}$ in this case is,

$$
\lambda_{\mathrm{TeV}}=\frac{16 \pi^{2}}{N^{2}} \epsilon^{3 / 2} v_{\mathrm{ir}}^{2}
$$


which leads to the following parametric form for the bounce action [20],

$$
\frac{S_{3}}{T} \sim \frac{N^{7 / 2}}{\epsilon^{9 / 8} v_{\mathrm{ir}}^{3 / 2}} \frac{T_{c}}{T}\left(1-\left(\frac{T}{T_{c}}\right)^{4}\right)^{-2} .
$$

The action is not only enhanced by the factor of $N^{2}$, but also by the small quartic coupling of the radion, which increases the dependence on $N$ to $N^{7 / 2}$. There is an additional enhancement by $1 / \epsilon$, related to the fact that the scale symmetry violation at $\mu_{\mathrm{TeV}}$ is parametrised by $\epsilon$. The exact power of $\epsilon$ that appears can depend on the implementation of the GW mechanism [20, 24, 34, 38].

More generally, we can see that the action is enhanced for small scale symmetry violation encoded in $\beta_{\lambda} \ll 1$. The zero temperature minimum is determined by the running quartic, $\lambda(g(\mu))$,

$$
\partial V(\mu) / \partial \mu=\left[4 \lambda\left(g\left(\mu_{\mathrm{TeV}}\right)\right)+\beta_{\lambda}\left(g\left(\mu_{\mathrm{TeV}}\right)\right)\right] \mu^{3}=0 .
$$

Thus for a nearly scale invariant theory at $\mu_{\mathrm{TeV}}, \lambda_{\mathrm{TeV}}$ will be generically small.

If the transition is not prompt, then it will then take place in the supercooled regime, $T \ll T_{c}$. In the case where there is a barrier in the radion potential between $\mu_{\mathrm{TeV}}$ and $\mu \sim 0$, the bounce configuration is essentially the same as the zero temperature tunnelling, and has an $\mathrm{O}(4)$ symmetric bounce action [20, 24]

$$
S_{4} \sim \frac{N^{2}}{16 \pi^{2} \lambda_{\mathrm{TeV}}}
$$

As before, the explicit factor $N^{2}$ appears. Again, we see that in the case of the simplest $\mathrm{RS}+\mathrm{GW}$ model above, the parametric dependences are even stronger,

$$
S_{4} \sim \frac{N^{4}}{(4 \pi)^{4} \epsilon^{3 / 2} v_{\text {ir }}^{2}} .
$$

If there is no barrier between $\mu \sim 0$ and $\mu=\mu_{\mathrm{TeV}}$, then the "release point" for the radion field can be very small $\mu \sim T$ even for supercooled transition, so the smallest bounce action is still obtained by an $\mathrm{O}(3)$ symmetric bounce. For example, in the case where conformal symmetry is restored in the IR $(\epsilon>0$ for the GW field) [34], the radion potential near the origin is $V \sim \lambda(0) \mu^{4}$. The bounce action is then the $T \ll T_{c}$ limit of equation (2.15),

$$
\frac{S_{3}}{T} \sim \frac{N^{2}}{8[\lambda(0)]^{3 / 4}}
$$

which is no longer suppressed by the small parameter $\epsilon$. In the case where $\epsilon \lambda_{\mathrm{TeV}}$ is not parametrically small, radion dynamics no longer suffice to estimate the bounce. However, it may still be possible to estimate the bounce in the $5 \mathrm{D}$ effective theory [38] and is found to be $\mathcal{O}\left(N^{2}\right)$.

When $\epsilon<0$, the GW field profile grows towards the IR. Consequently, the higher order terms in the GW potential might become important and the approximate conformality 
$\partial_{\log \mu} g(\mu) \sim \epsilon$ might be broken as we approach $\mu \lesssim \mu_{\mathrm{TeV}}$. In such cases the enhancement of the bounce action by $1 / \epsilon$ will be absent, even though the EW/Planck hierarchy is set by small epsilon. This can be explicitly seen in explicit holographic constructions [39, 61], or in RS models with more general stabilisation mechanisms [24-35, 37, 38].

We see from the examples above that while the details of the bounce action depend on the actual theory, it takes the form $S_{b} \simeq N^{2} / \lambda$ in each case, with $\lambda \lesssim 1$. This has farreaching consequences for early universe cosmology. Either the universe is required to be reheated to temperatures lower than the confinement scale, or there is a strong constraint on the maximal $N$ allowed.

If the rate of tunnelling is smaller than Hubble, the universe will get stuck in the false vacuum [62]. Since the true vacuum at zero temperature is assumed to have a (nearly) vanishing cosmological constant, the deconfined vacuum has a large positive cosmological constant $C \sim N^{2} T_{c}^{4}$ at low temperatures and starts to inflate with $H \simeq N T_{c}^{2} / M_{\mathrm{pl}}$. In a Hubble volume, the probability of completing the phase transition within a Hubble time is,

$$
P=\Gamma / H^{4}
$$

If $P \ll 1$, then the universe eternally inflates. This gives us a bound on $N$,

$$
N \lesssim 2 \sqrt{\lambda \log \frac{M_{\mathrm{pl}}}{T_{c}}}
$$

We have replaced the inverse critical radius by $T_{c}$; unless the bubble size is exponentially smaller, this is a reasonable approximation. In many models considered above, $\lambda$ is parametrically small. For the RS+GW model above, $S_{b}$ is enhanced both by $N$ as well as $1 / \epsilon$, making it impossible to satisfy the constraint above. Even without these enhancements, the calculations above assume dilaton dominance, which requires $\lambda_{\mathrm{TeV}} \ll 1$ [34]. Therefore, it is hard to get $S_{b} \lesssim N^{2}$ in a controlled approximation. This translates into a bound $N \lesssim 12$ for $T_{c} \sim 1 \mathrm{TeV}$. From equation (2.2), we see that the hierarchy between the 5D Planck scale and the AdS curvature is $\left(M_{5} / k\right) \lesssim 1$. This lack of hierarchy makes the 5D effective gravitational theory very delicate.

One avenue to evade this cosmological bound is to avoid reheating the universe above the $\mathrm{TeV}$ scale. This may require a more intricate inflationary mechanism, as well as solutions to baryogenesis at the electroweak scale or below $[29,30,42]$. In the next section we outline the avoided deconfinement mechanism, where the GW stabilisation of the radion is temperature dependent and the IR brane is stabilised at arbitrarily high temperatures. This allows for parametrically large hierarchies between $M_{5}$ and $k$, and an early cosmology without a stringent restriction on the reheat temperature.

\section{$3 \quad 5 \mathrm{D}$ model for avoided deconfinement}

In this section we modify the RS model with GW field $(\Phi)$ by including extra scalars localised to the IR brane. ${ }^{1}$ Given a suitable set of parameters, the effect of this will be

\footnotetext{
${ }^{1}$ Localised fields on the IR brane may be required to arise from corresponding bulk modes with masses below the 5D cutoff [63]. These bulk modes will then have an associated tower of KK states, but this detail will not affect our discussion.
} 
to realise a model where the new scalars provide a metastable minimum for the radion at high temperature, avoiding the formation of the AdS-S black hole.

We make a simple modification to the RS model described in equation (2.6) by adding scalar field(s) $S$ to the IR brane. The action is:

$$
S=S_{\mathrm{bulk}, \mathrm{RS}}+S_{\mathrm{uv}, \mathrm{RS}}+S_{\mathrm{ir}, \mathrm{RS}}+S_{\mathrm{ir}, \mathrm{AD}}
$$

where $S_{\text {bulk,RS }}$ and $S_{\mathrm{uv} / \mathrm{ir}, \mathrm{RS}}$ are the RS model bulk and brane actions which are unchanged from their definition in equations (2.4), (2.5), and (2.6). We continue to choose the detuning parameter $\delta \Lambda_{\mathrm{ir}}=0$. As usual, this simplifying assumption can be relaxed. The modified IR brane action, $S_{\mathrm{ir}, \mathrm{AD}}$ includes $N_{s}$ real scalars $S$ localised to the brane. The additional terms in the IR brane action is

$$
S_{\mathrm{ir}, \mathrm{AD}}=k^{4} \int_{\rho=\rho_{\mathrm{ir}}} d^{4} x \sqrt{-g_{\mathrm{ir}}} \sum_{i=1}^{N_{s}}\left[\frac{1}{2 k^{2}} g_{\mathrm{ir}}^{\mu \nu} \partial_{\mu} S_{i} \partial_{\nu} S_{i}-\frac{\lambda_{s}}{4}\left(S_{i}^{2}-v_{s}^{2}\right)^{2}-\frac{\gamma}{6} S_{i}^{3}\right],
$$

where we have explicitly included factors of $k$ so that the parameters $\lambda_{s}, \gamma, v_{s}$ as well as the field $S$ are dimensionless. We will suppress the index $i$ on $S$ for notational simplicity. In order for the potential to remain bounded from below, the coupling $\lambda_{s}$ must be positive. The value of the masses and quartic couplings of each field $S$ do not have to be equal, but for simplicity we will take them to be the same. For unequal couplings our results below can be reinterpreted using statistical averages over the $S$ ensemble. Each $S$ has an approximate $Z_{2}$ symmetry that is spontaneously broken at zero temperature. The coupling $\gamma$ that weakly breaks the $Z_{2}$ symmetry for each $S$ is introduced to avoid domain wall problems, and can be very small in a technically natural way.

Before presenting the consequences of adding these extra scalars, we summarize the choice of parameters for which our approximations are under theoretical control. The primary goal of the $\mathrm{AD}$ setup is to generate a classically stable minimum for the radion at high temperatures for arbitrary $N$, therefore avoiding a confinement phase transition entirely and putting the large- $N$ approximation on a firmer footing. It is then worth highlighting the validity of the large- $N$ expansion, especially in light of adding extra matter on the IR brane. Requiring the gravitational loop counting parameter to be small, $N_{\text {species }} / N^{2} \lesssim 1$, restricts the number of scalars we can add. As we show below, the AD mechanism does require $N_{s}>1$ to operate. However, in order to obtain a classically stabilized radion at high temperatures, $N_{s}$ can be parametrically smaller than $N^{2}$. In this case, the black hole phase does have a lower free energy, but the tunneling rate from the $\mathrm{AD}$ phase to the black hole phase is exponentially suppressed by a tunnelling exponent of order $\sim N^{3}$. We present an estimate of the tunneling rate in appendix A. Thus, the parameter $N$ can be taken arbitrarily large while keeping other parameters in our model fixed, ensuring that the $1 / N$ expansion is well under control.

In order to understand the other parametric scalings in the Lagrangian, it is useful to characterize the cosmological history of the $\mathrm{AD}$ model by the following three scales: 
1. the temperature $T_{s}$ at which the scalars $S$ undergo a (crossover) phase transition;

2. the temperature $T_{c}$ at which the $\mathrm{AD}$ construction begins to take effect and the position of the IR brane begins to vary with temperature; and

3. the zero-temperature radion vev, $\mu_{\mathrm{TeV}}$.

The AD model requires the hierarchy $T_{s}<T_{c}$, due to the fact that the addition of the new scalars $S$ only generates the desired finite-temperature effects in the symmetric phase. At temperatures $T>T_{c}$ the position of the IR brane is moved from the GW minimum due to thermal effects. As mentioned above, in this temperature range the confined phase is metastable, in contrast to the usual RS model where the confined phase becomes classically unstable at high temperature. Another condition on the parameter space comes from the requirement that the IR brane is stabilized at a radius where the local temperature is small enough such that the backreaction on the bulk geometry is small. At the confinement scale, this condition implies

$$
T_{c}<\frac{\mu_{\mathrm{TeV}}}{\pi},
$$

This condition becomes stronger logarithmically in temperature, and can fail at very high temperatures, which sets a maximum temperature $T_{\max }$ for the $\mathrm{AD}$ mechanism to operate. As we explain in further detail in section 3.2, this leads to the following condition on the parameters of the model:

$$
1>\frac{\pi T_{c}}{\mu_{\mathrm{TeV}}}>\sqrt{\frac{6}{N_{s}}} \frac{m_{\varphi}}{m_{s}}>v_{s}
$$

where $m_{\varphi}, m_{s}$ are the masses of the radion and scalar $(s)$ fluctuations at zero temperature. Since the mass of the radion $m_{\varphi}$ cannot be too small phenomenologically, this leads us to require a moderately large number of scalar fields $N_{s}$ for the AD model to work.

\subsection{Finite temperature effective potential for the radion}

We work in a regime where the local $5 \mathrm{D}$ temperature remains below $\left(M_{5}^{3} k^{2}\right)^{1 / 5}$ everywhere in the 5th dimension, so that the finite temperature effects have a negligible backreaction effect on the bulk geometry. At any temperature $T$ and the position of the IR brane $\rho_{\text {ir }}(T)$, we can solve the equations of motion for the GW field on the background RS metric with the same boundary conditions, $\Phi\left(\rho_{\text {ir } / \mathrm{uv}}(T)\right)=k^{3 / 2} v_{\text {ir } / \mathrm{uv}}$. The bulk solution is,

$$
\Phi(\rho)=A \rho^{-4-\epsilon}+B \rho^{\epsilon}
$$

where $A, B$ are fixed by the boundary conditions. As above, $\epsilon \approx m_{\Phi}^{2} /\left(4 k^{2}\right)$, which we take to be positive. We expand in fluctuations around this classical solution with the size of the extra dimension equal to $\rho_{\text {ir }}(T)$. We decompose the bulk fluctuations into Kaluza-Klein modes and integrate over the $4 \mathrm{D}$ modes to derive the finite temperature effective potential.

The temperature-dependent effective potential can be broken up into the tree-level potential and the one-loop potential $[64,65]$ :

$$
V_{\text {eff }}(T)=V_{\text {tree }}+\Delta V_{1}^{\mathrm{CW}}+\Delta V_{1}^{T}(T)
$$


where we have separated the 1-loop contribution into a piece that goes to 0 at zero temperature $\Delta V_{1}^{T}(0)=0$. The zero temperature Coleman-Weinberg potential, $\Delta V_{1}^{\mathrm{CW}}$ includes the usual UV-divergences one would encounter in these calculations. The tree-level $4 \mathrm{D}$ action is obtained as above by integrating the classical solution over the extra dimension. The potential is given by,

$$
V_{\text {tree }}\left(\mu, S_{i}\right)=\mu^{4}\left[(4+2 \epsilon)\left(v_{\text {ir }}-v_{\text {uv }}(\mu / k)^{\epsilon}\right)^{2}-\epsilon v_{\text {ir }}^{2}+\sum_{i=1}^{N_{s}}\left(\frac{\lambda_{s}}{4}\left(S_{i}^{2}-v_{s}^{2}\right)^{2}+\frac{\gamma}{6} S_{i}^{3}\right)\right]
$$

where we have used $\mu=k^{2} \rho_{\text {ir }}(T)$. We have also suppressed the $T$-dependence in the notation.

The one-loop contribution is obtained by integrating over the fluctuations. The finite temperature contribution to the potential depends on the effective mass of the fluctuations around the classical solution. The relevant particles in our case are the radion, the new scalars and the SM fields. Including the kinetic term for the radion $[58,66]$ and the scalars, we find the following action for the fluctuations:

$$
S=\int d^{4} x\left[\mathcal{L}_{\mathrm{SM}}+\frac{1}{2}(\partial \varphi)^{2}+\frac{1}{2}\left(\partial s_{i}\right)^{2}-V_{\text {tree }}\left(\mu\left(1+\frac{\varphi}{F_{\varphi}}\right), S_{i}+\frac{s_{i}}{\mu}\right)\right]
$$

We have introduced the canonically normalized fluctuations for the radion $\varphi$ and the scalars $s$. The radion decay constant

$$
F_{\varphi}=\frac{N}{2 \sqrt{2} \pi} \mu .
$$

The indices in the kinetic term are now contracted with the 4d Minkowski metric.

The field-dependent masses of these particles are defined as the second derivative of the potential w.r.t. the corresponding field. The SM particle masses and the radion and $s$ masses all scale with $\mu$. For a large number of scalars $N_{s} \gg 1$, the thermal potential is dominated by loops of $s_{i}{ }^{2}$ The field-dependent masses of $s_{i}$ are,

$$
m_{s, i}^{2}=\mu^{2}\left[-\lambda_{s} v_{s}^{2}+3 \lambda_{s} S_{i}^{2}+\gamma S_{i}\right]
$$

The other modes in the spectrum are the KK modes of the graviton, the GW field and other fields in the bulk. The mass of the $n$th KK mode is approximated by [67]:

$$
m_{n} \simeq\left(n+\frac{2+\epsilon}{2}-\frac{3}{4}\right) \pi \mu .
$$

The $\rho$-coordinate of the would-be AdS-S horizon is $\rho_{h}=\pi T / k^{2}$. Therefore, the condition that the IR brane is stabilised outside the AdS-S horizon, $\rho_{\text {ir }}(T)>\rho_{h}$ implies that the higher KK modes are not excited at any $T$, and can be safely neglected in the thermal potential.

\footnotetext{
${ }^{2}$ Note that since the SM contribution to the $\mu$ potential is proportional the mass of the SM field, only the contributions from $t, W, Z, h$ are sizeable.
} 
The Coleman-Weinberg potential is given by,

$$
V_{1}^{\mathrm{CW}}\left(\mu, S_{i}\right)=\sum_{i=1}^{N_{s}} \frac{1}{64 \pi^{2}} m_{s, i}^{4}\left(\log \left[\frac{m_{s, i}^{2}}{\mu_{R}^{2}}\right]-\frac{3}{2}\right)
$$

where $\mu_{R}$ is a renormalisation scale. A convenient choice of the renormalisation scale for the dynamics on the IR brane is $\mu$ itself [68]. With this choice, we do not generate large hierarchies of scale on the IR brane and the one-loop corrections at zero-temperature stay small. We have included all terms allowed by the scale symmetry of the radion and the $Z_{2}$ symmetries in the $s$-sector, as well as leading terms violating these symmetries parameterized by the small parameters $\epsilon, \gamma$. Thus, the higher order terms can be safely neglected and we will simply absorb the UV divergent pieces into a redefinition of couplings and masses as renormalized quantities.

The finite-temperature one-loop contributions from $s_{i}$ are

$$
\begin{aligned}
\Delta V_{1}^{T}\left(\mu(T), S_{i}(T), T\right) & =\sum_{i=1}^{N_{s}} \frac{T^{4}}{2 \pi^{2}} \int d k k^{2} \log \left[1-\exp \left(-\sqrt{k^{2}+\frac{m_{s, i}^{2}\left(\mu, S_{i}\right)}{T^{2}}}\right)\right] \\
& \equiv \sum_{i=1}^{N_{s}} \frac{T^{4}}{2 \pi^{2}} J_{b}\left(\frac{m_{s, i}^{2}(\mu, S)}{T^{2}}\right)
\end{aligned}
$$

We approximate the thermal function $J_{b}$ by assuming that $m_{s}(T) \ll T$. At high temperature the thermal function can be approximated as,

$$
J_{b}\left(y^{2}\right) \approx-\frac{\pi^{4}}{45}+\frac{\pi^{2}}{12} y^{2}-\frac{\pi}{6} y^{3}-\frac{1}{32} y^{4}\left(\log \frac{y^{2}}{\pi^{2}}+2 \gamma_{E}-\frac{3}{2}\right) .
$$

where $\gamma_{E}$ is the Euler-Mascheroni constant. The field-dependent log pieces cancel between the Coleman-Weinberg terms and the thermal corrections. The renormalisation scale $\mu_{R} \simeq$ $\mu$ scales with the temperature (as we show below), and hence we do not get any enhanced large-log pieces, and we can safely ignore the terms of $\mathcal{O}\left(y^{4}\right)$. Then,

$$
\begin{aligned}
\Delta V_{1}^{T}(\mu, S, T) \simeq & \frac{N_{s}}{24} T^{2} \mu^{2}\left(-\lambda_{s} v_{s}^{2}+3 \lambda_{s} S^{2}+\gamma S\right) \\
& -\frac{N_{s}}{12 \pi} T \mu^{3}\left(-\lambda_{s} v_{s}^{2}+3 \lambda_{s} S^{2}+\gamma S+\lambda_{s} \frac{T^{2}}{4 \mu^{2}}\right)^{3 / 2}
\end{aligned}
$$

where we have used the fact that each of the scalar vevs $S_{i}=S$ when $\left\{\lambda_{s}, v_{s}, \gamma\right\}$ are taken to be the same for each $s_{i}$. The extra term involving $T^{2}$ in the second term above is a result of performing the leading daisy resummation, where we replace the field-dependent mass $m_{s, i}^{2}$ by $m_{s, i}^{2}+\Pi_{i}$ in equation (3.14), with $\Pi_{i}$ the leading temperature contribution to the one-loop thermal mass.

Thus, we see that at high temperatures $T>T_{s} \simeq \frac{1}{2} v_{s} \mu_{\mathrm{TeV}}$, thermal effects drive to restore the (approximate) $Z_{2}$ symmetry in $S$, so that $\langle S\rangle \ll 1$. This generates a tachyonic direction for $\mu$, providing a finite temperature stabilization. As the universe cools, the $S$ symmetry gets broken, and the radion settles down close to its zero temperature minimum dictated by the GW part of the potential. The thermal potential can be minimized numerically, and for a range of parameters the radion remains stabilized outside the would-be AdS-S horizon at high temperatures. 


\subsection{High temperature radion stabilization}

The minimum of the radion potential can be simply approximated in two distinct regimes:

$$
\mu(T)=\left\{\begin{array}{lr}
\mu_{\mathrm{TeV}} & T<T_{c} \\
\mu_{\mathrm{TeV}}\left(\frac{T}{c T_{c}}\right)^{\frac{1}{1+\epsilon}} & T \gg T_{c}
\end{array}\right.
$$

where the constant $c$ is given by:

$$
c^{2}=\frac{4 v_{\mathrm{uv}}^{2}}{\epsilon^{3 / 2} v_{\mathrm{ir}}^{2}}\left(\frac{\mu_{\mathrm{TeV}}}{k}\right)^{2 \epsilon}
$$

The zero temperature value of the radion minimum $\mu_{\mathrm{TeV}}$ is well approximated by equation (2.8), up to an $\mathcal{O}\left(\gamma v_{s}^{3}\right)$ correction to the $\mu^{4}$ co-efficient which is a result of the potential for $S$ not vanishing at the zero-temperature minimum. The transition temperature $T_{c}$ is the temperature at which the radion starts to move and is given by,

$$
T_{c}^{2} \simeq \frac{6}{N_{s}} \frac{m_{\varphi}^{2}}{m_{s}^{2}} \mu_{\mathrm{TeV}}^{2}=\frac{24 \mu_{\mathrm{TeV}}^{2}}{N_{s}\left(\lambda_{s} v_{s}^{2}\right)} \epsilon^{3 / 2} v_{\mathrm{ir}}^{2}
$$

where $m_{\varphi}, m_{s}$ here are the zero-temperature masses for $\varphi, s$. Since $\mu(T) / T$ is slowly growing, there is a maximum temperature $T_{\max }$ beyond which the IR brane would fall behind the horizon,

$$
T_{\max } \sim \mu\left(T_{\max }\right) / \pi \Rightarrow T_{\max }=\frac{\mu_{\mathrm{TeV}}}{\pi}\left(\frac{\mu_{\mathrm{TeV}}}{\pi c T_{c}}\right)^{1 / \epsilon}
$$

This sets a (mild) bound on the reheat temperature of the universe. This is at an exponentially high scale for $\delta \equiv \pi T_{c} / \mu_{\mathrm{TeV}} \ll 1 / c$. The $\mathrm{AD}$ transition temperature should also be higher than the $Z_{2}$ symmetry restoration temperature, $T_{c}>T_{s}$. These two requirements give us the following inequalities on our parameter space,

$$
\delta>\sqrt{\frac{6}{N_{s}}} \frac{m_{\varphi}}{m_{s}}>v_{s}
$$

For illustration, we choose the following benchmark values ${ }^{3}$

$$
\begin{aligned}
& \left\{k=6 \times 10^{16} \mathrm{GeV}, \epsilon=4.13 \times 10^{-2}, v_{\mathrm{uv}}=10^{-3}, v_{\mathrm{ir}}=3 \times 10^{-4},\right. \\
& \left.N_{s}=100, \lambda_{s}=1, v_{s}=2 \times 10^{-3}, \gamma=-10^{-8}\right\},
\end{aligned}
$$

and find the following parameters

$$
\begin{aligned}
\mu_{\mathrm{TeV}} & \simeq 1.8 \times 10^{-12} k \simeq 100 \mathrm{TeV} \\
T_{c} & \simeq 700 \mathrm{GeV} .
\end{aligned}
$$

The maximum temperature $T_{\max }$ for this case is around $5 \times 10^{11} \mathrm{GeV}$. The temperature evolution of various scales in this benchmark is illustrated in figure 2 .

\footnotetext{
${ }^{3}$ The value of $\epsilon$ was chosen such that $\mu_{\mathrm{TeV}} \simeq 100 \mathrm{TeV}$. A more generic choice works equally well.
} 

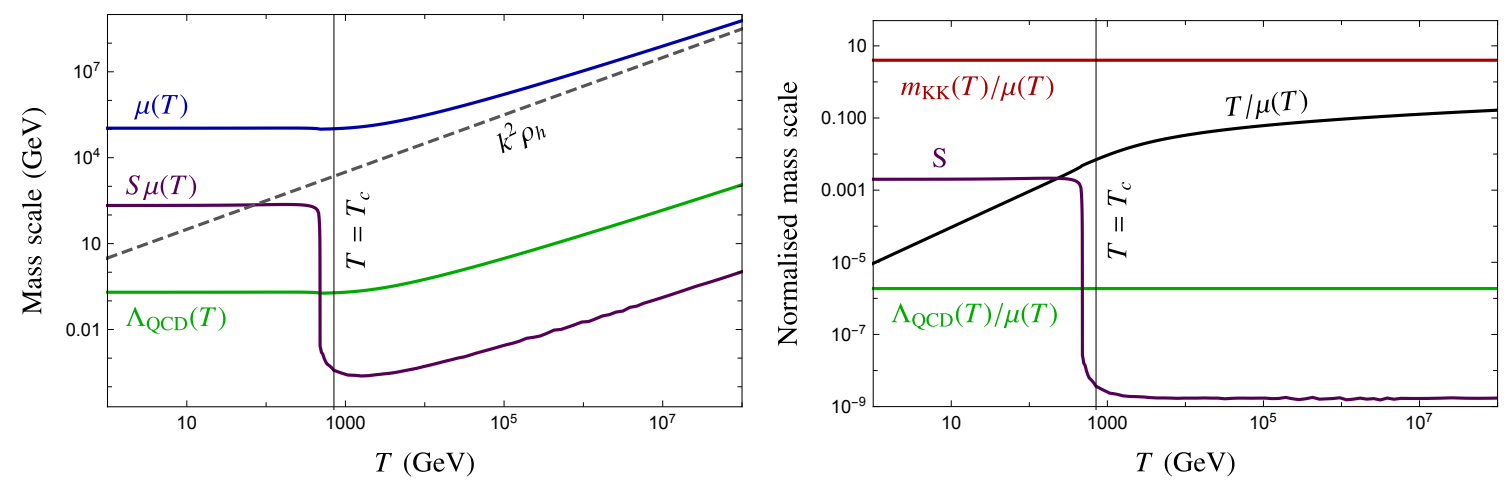

Figure 2. Plots showing the dependence of mass scales in the theory with temperature, for parameters in equation (3.22). The left plot shows the radion expectation value $(\mu)$, the scalar expectation value $(S \mu)$ and the QCD scale, while the second plot shows the same quantities and the KK scale with the dependence on the radion factored out. The vertical line shows the critical temperature $T_{c} \sim 700 \mathrm{GeV}$. The dashed black line in the first plot shows the horizon location $k^{2} \rho_{h}$ as a function of temperature.

Comparing the AD model to the usual RS model, the high temperature behaviour is vastly different. In the RS model, as we move to high temperature, thermal effects drive the IR brane towards the AdS boundary, where it eventually collapses to form an AdS-S black hole [69]. The model then remains in the black hole phase until tunnelling to the RS phase through a first order phase transition which is highly suppressed in calculable models, as was shown in section 2. In contrast, the model presented here describes a situation where the IR brane is stabilised closer to the UV end of the warped direction at high temperatures, then falls approximately linearly with temperature into the IR before stabilising at a constant value deep in the IR as in the RS case. It should be noted that at very high temperatures, the black hole phase can still have a lower free energy than the AD phase, but the IR brane remains meta-stabilised. The only way the system can transition to the black hole phase is through a first order phase transition which is exponentially suppressed.

The AD model also introduces a novel temperature dependence of the mass scales on the IR brane, which scale linearly with the radion. Assuming that the SM is confined to the IR brane, the left panel of figure 2 shows the dependence of the QCD scale with temperature in the AD model. The other dimensional parameters of the SM, such as the electroweak vacuum value, exhibit a similar scaling above the critical temperature, $T_{c}$, of the theory. Above $T_{c}$ the temperature only increases marginally relative to the other scales of the theory. This leads to the KK modes being frozen out to arbitrarily high temperature, as shown in the right panel of figure 2. A similar behaviour would occur for any other scales of the theory which are above $T_{c}$ - they are frozen out to much higher temperatures than in the usual RS model. In section 5 we discuss this behaviour and its potential implications for BSM phenomenology in more detail. 


\section{Low temperature phenomenology}

In this section we show we study the constraints on the avoided deconfinement model from collider results and ALP searches. For simplicity we have assumed that the SM is confined to the IR brane. More realistic models typically have some or all SM fields propagating in the bulk, which can offer an explanation of the hierarchical Yukawa couplings in the SM [67, 70, 71] but also lead to new constraints from flavour-violating processes [72, 73]. The constraints on the RS model have been well-studied. Here we will focus on the new features that are required for the $\mathrm{AD}$ mechanism to work.

The qualitative features that the mechanism requires can be estimated using the inequality in equation (3.4). For $\delta \ll 1$, we need,

$$
\begin{aligned}
\frac{m_{s}}{\mu_{\mathrm{TeV}}} & \simeq v_{s}<\delta \\
\frac{m_{\varphi}}{\mu_{\mathrm{TeV}}} & <\delta^{2} \sqrt{N_{s}} .
\end{aligned}
$$

Experimental constraints on a very light radion will lead us to require a large number of scalars, $N_{s} \gg 1$, with masses below the confinement scale.

In section 4.1 we write down the effective Lagrangian - the relevant degrees of freedom being the SM fields, the radion $\varphi$, and the AD scalar(s) $s$. We ignore higher dimensional operators which arise from integrating out KK modes and $1 / N$ suppressed stringy corrections, taking these to be negligible. In section 4.2 we then describe the dominant experimental constraints in different regions of parameter space.

\subsection{Effective Lagrangian at zero temperature}

The tree-level interactions of the radion $\varphi$ with the SM fields can be written compactly by the replacing the mass terms in the SM by a $\varphi$ dependent mass,

$$
\mathcal{L}^{\text {(tree) }}\left[m_{i}\right] \rightarrow \mathcal{L}_{\text {int }}^{\text {(tree) }}\left[m_{i}\left(1+\frac{\varphi}{F_{\varphi}}\right)\right]
$$

where we the decay constant $F_{\varphi}$ was defined in equation (3.9). This produces Yukawa-like interactions of $\varphi$ with the fermions, as well as trilinear and quartic couplings with the Higgs and the gauge bosons. This form of the radion potential is dictated by the AdS isometries (and hence is valid in the limit of $\epsilon \ll 1$ ). The self-interaction terms for the radion are generated by the GW mechanism,

$$
\mathcal{L}_{\text {radion }}=\frac{1}{2} m_{\varphi}^{2} \varphi^{2}\left(1+\frac{5}{3} \frac{\varphi}{F_{\varphi}}+\frac{11}{12} \frac{\varphi^{2}}{F_{\varphi}^{2}}\right)
$$

where we have only kept terms to leading order in $\epsilon$. Finally, the scalars $s$ interact with the SM through the radion portal.

$$
\mathcal{L}_{\mathrm{s}}^{\text {(tree) }}=\lambda_{s} v_{s}^{2} \mu_{\mathrm{TeV}}^{2} s^{2}\left(1+\frac{\varphi}{F_{\varphi}}\right)^{2}+\lambda_{s} v_{s} \mu_{\mathrm{TeV}} s^{3}\left(1+\frac{\varphi}{F_{\varphi}}\right)+\frac{1}{4} \lambda_{s} s^{4}+\mathcal{O}(\gamma)
$$


The terms suppressed by the explicit $Z_{2}$ violating coupling $\gamma$ are assumed to be very small, and do not contribute significantly to the zero-temperature phenomenology. Notice that we do not generate terms of the form of $\varphi$-s mixing, or $s \varphi^{3}$. The classical solution sets the linear term in $s$ to zero, and the $\varphi$ field coupling as $(1+\varphi)$ then does not have a linear coupling to $s$ around the vacuum. A small Higgs portal coupling of the form $\kappa s H^{\dagger} H$ can be added in order for $s$ to be able to decay safely before BBN.

At loop level, there are also induced couplings between the EM and QCD field strengths proportional to their $\beta$-functions:

$$
\mathcal{L}_{\text {int }}^{(1-\text { loop })} \supset \frac{\alpha_{\mathrm{EM}}}{8 \pi F_{\varphi}} b_{\mathrm{EM}} \varphi F_{\alpha \beta} F^{\alpha \beta}+\frac{\alpha_{s}}{8 \pi F_{\varphi}} b_{\mathrm{G}} \varphi G_{\alpha \beta}^{a} G^{a \alpha \beta},
$$

with the dominant contributions to these terms coming from quark and $W$-boson loops. In the case where the $\mathrm{SM}$ is confined to the IR brane, $b_{\mathrm{EM}}=11 / 3, b_{\mathrm{G}}=-\frac{11}{3} N_{c}+2 n / 3$, where $n$ is the number of quarks lighter than the radion [74].

\subsection{Experimental constraints}

The low energy phenomenology of the model is largely determined by the physical masses of the radion and $s$ fields, as well as the KK scale. These are related to the fundamental parameters of the model by:

$$
\begin{aligned}
m_{\mathrm{KK}} & \simeq \frac{5 \pi}{4} \mu_{\mathrm{TeV}} \\
m_{\varphi} & =2 \sqrt{2} v \epsilon^{3 / 4} \mu_{\mathrm{TeV}} \\
m_{s} & =\sqrt{2 \lambda_{s}} v_{s} \mu_{\mathrm{TeV}}
\end{aligned}
$$

Collider searches limit the KK scale in RS models to be above $m_{\mathrm{KK}} \gtrsim 4.25 \mathrm{TeV}[75,76]$, requiring the KK resonances to be out of the kinematic reach of current colliders. Due to the approximate shift symmetry of the GW field (broken only by the small parameter $\epsilon$ ), the radion is parametrically lighter than the KK scale. The AD scalar masses are similarly suppressed, with $m_{s}$ proportional to the combination $\left(\lambda_{s} v_{s}^{2}\right)^{1 / 2}$, which is chosen to be small for the $s$ phase transition to happen well before the deconfinement transition. Therefore, the radion and the AD scalars can be kinematically accessible at colliders [77], however their couplings to the SM are suppressed by $\mu_{\mathrm{TeV}}$. Collider constraints translate into a bound $\mu_{\mathrm{TeV}} \gtrsim 2 \mathrm{TeV}$ [74], which is weaker than direct bounds on the KK scale.

If the radion mass is below the $\mathrm{GeV}$ scale, bounds on the $\varphi \gamma \gamma$ coupling from supernova cooling, ${ }^{4}$ cosmology and beam dump experiments can give the strongest bounds on the model. These limits have been derived for axion-like particles [79-81], which translate into a bound

$$
F_{\varphi} \gtrsim 4.25 \times 10^{7} \mathrm{TeV}
$$

\footnotetext{
${ }^{4}$ Whether supernova bounds on the radion coupling apply depends on the radion coupling to nucleons [78]. In the case where the SM quarks and gluons are on the IR brane, this coupling is too large for the radion to contribute significantly to supernova cooling.
} 
For a heavier radion $m_{\varphi}>1 \mathrm{GeV}$, these constraints are no longer applicable. The radion mass will be above a $\mathrm{GeV}$ for parameters,

$$
v \epsilon^{3 / 4}>7 \times 10^{-5}\left(\frac{\mu_{\mathrm{TeV}}}{5 \mathrm{TeV}}\right)^{-1} .
$$

The AD scalars can couple to the SM fields through the radion portal. For light AD scalars, the couplings to photons/gluons generated at higher loop order might still provide significant constraints. If the $\mathrm{AD}$ scalars are above the $1 \mathrm{GeV}$ scale, these constraints are also absent.

\section{Cosmology}

The mechanism of avoided deconfinement has dramatic implications for early universe cosmology. The main departure from a standard cosmology is due to the scaling of the radion expectation value with temperature. This leads to the interesting consequence that while the universe may be reheated to a very high temperature (exciting heavy fields on the UV brane, for instance), from the IR brane point-of-view, the cosmology resembles a low-reheat cosmology. We aim to highlight some of the applications of the AD model to cosmology, but leave a detailed study of these implications for future work.

Figure 2 shows the characteristic dependence on temperature of dimensionful parameters on the IR brane. In particular, $m_{K K}>T$ to arbitrarily high temperature, as required by the condition that the IR brane be stabilised outside the horizon at a given temperature. Therefore, KK modes play no role in early universe cosmology from the point of view of the IR brane. In the high temperature regime $T>T_{c}$, the radion expectation value scales with temperature as:

$$
\mu(T) \propto T^{\frac{1}{1+\epsilon}}
$$

This introduces a scaling of the other dimensionful quantities of the theory with $T$. The KK scale, the Higgs mass parameter and the QCD scale and are all proportional to $\mu(T),{ }^{5}$ which means that the ratio of the temperature to these mass scales (denoted $\Lambda$ ) varies with $T$ as:

$$
\frac{T}{\Lambda} \propto T^{\frac{\epsilon}{1+\epsilon}}
$$

The consequence of this scaling is that the ratio $T / \Lambda$ may reach unity at significantly higher temperatures than is the case in standard RS cosmology. For example, as we show below, if the critical temperature $T_{c}$ is below the electroweak symmetry breaking scale, then the electroweak symmetry restoration phase transition may occur at much higher temperatures than in the usual case, or never occur at all.

\footnotetext{
${ }^{5}$ More generally, $\Lambda_{\mathrm{QCD}} \propto(\mu(T))^{n}$, where $n=1$ is true for the case where the SM is confined to the IR brane, $n<1$ if some of the SM quark fields are bulk fields.
} 


\subsection{Electroweak phase transition}

In this section we show that the electroweak phase transition can occur in the avoided deconfinement phase at temperatures much higher than the weak scale. To illustrate some of these effects, we use a new set of parameters, with:

$$
v_{\text {ir }}=1.5 \times 10^{-4}, v_{\mathrm{uv}}=7.5 \times 10^{-4}, \epsilon=0.05
$$

and all other parameters as in equation (3.22), which leads to a radion stabilised at $\mu_{\mathrm{TeV}}=4.73 \mathrm{TeV}$ at zero temperature. We note that with these parameters, the model in its simplest form does not satisfy the bound on the radion mass (4.10). We expect that a more complete model with additional breaking of scaling invariance can lead to an unsuppressed radion mass and a less severe bound than equation (4.10). This could happen, for example, through additional terms in the GW action [66], allowing more fields to propagate in the bulk, or by considering a more general geometry for the fifth dimension [39]. We will leave the detailed model building for future work.

The Higgs potential at finite temperature gets thermal corrections from the top Yukawa, gauge couplings and its quartic coupling. In addition, the Higgs mass parameter scales with $\mu(T)$. The Higgs thermal mass in the low- and high-temperature limits is given by:

$$
\begin{aligned}
& \mu_{h}^{2}\left(T>T_{c}\right)=T^{2}\left(-\lambda \frac{v_{\mathrm{ew}}^{2}}{c^{2} T_{c}^{2}}\left(\frac{c T_{c}}{T}\right)^{2 \epsilon}+\frac{\lambda_{t}^{2}}{4}+\frac{3 g^{2}}{16}+\frac{g^{\prime 2}}{16}+\frac{\lambda}{2}\right), \\
& \mu_{h}^{2}\left(T<T_{c}\right)=-\lambda v_{\mathrm{ew}}^{2}+T^{2}\left(\frac{\lambda_{t}^{2}}{4}+\frac{3 g^{2}}{16}+\frac{g^{\prime 2}}{16}+\frac{\lambda}{2}\right) .
\end{aligned}
$$

where $v_{\text {ew }} \simeq 246 \mathrm{GeV}$. The electroweak phase transition (EWPT) happens at the temperature where the Higgs mass parameter become positive. This happens for $T<T_{c}$ if $T_{c}$ is above the electroweak scale, in which case there is no modification to the phase transition in comparison to the SM. However, if $T_{c}$ is below the electroweak scale, the EWPT will occur at a temperature:

$$
T_{*}=c T_{c}\left(\frac{T_{\mathrm{ew}}}{c T_{c}}\right)^{\frac{1}{\epsilon}}
$$

where $T_{\text {ew }}$ is the temperature of the EWPT in the SM. For small $\epsilon$, even a modest ratio $T_{\text {ew }} / T_{c}$ can lead to the EWPT occurring at a temperature which is orders of magnitude above the scale predicted by the SM. Figure 3 shows $v_{\text {ew }}$ as a function of temperature the two sets of parameters defined in equations (3.22) \& (5.3). For the second set of parameters the EWPT doesn't occur until the universe reaches a temperature of order $\sim 5 \times 10^{3} \mathrm{GeV}$.

A high temperature EWPT has been considered in refs. [41-44] in the context of electroweak baryogenesis. A primary motivation for these models is to avoid the bounds which result from introducing new sources of $\mathrm{CP}$ violation around the weak scale by having the EWPT occur at a temperature $T \gg v_{\text {ew }}$. This typically requires the introduction of a large number of fields coupled to the Higgs sector in order to significantly increase the temperature of the phase transition while satisfying collider bounds. In contrast, the AD model provides a mechanism in which the electroweak phase transition can occur at arbitrarily 


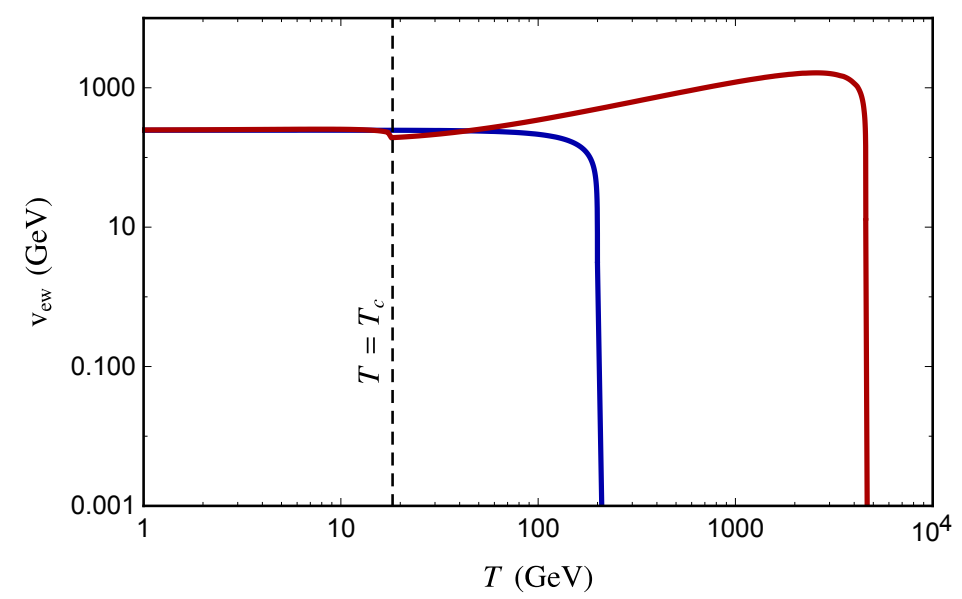

Figure 3. Higgs Higgs expectation value, $v_{\text {ew }}$, as a function of temperature for the parameters of equation (3.22) (blue) and equation (5.3) (red). The dashed line is the critical temperature, $T_{c}=18 \mathrm{GeV}$ for the choice of parameters in equation (5.3).

high temperatures due solely to the Higgs-radion interaction. However, the Higgs potential must still be modified to make the EWPT first order and introduce new sources of CP violation introduced in order to include a mechanism for electroweak baryogenesis in the AD framework. Further, even though the electroweak phase transition happens at much higher temperatures, the scales governing local physics on the IR brane also scale with $T$. Thus, if the CP violating operators are localized on the IR brane, their effect at $T=T_{*}$ will be the same as that at $T=T_{c} \lesssim v_{\text {ew }}$. Therefore, they will be subject to the very constraints the models of [41-44] were constructed to avoid. On the other hand, if the CP violating operators are on the UV brane/bulk, their effect does become much more important at higher temperatures. It will be interesting to construct and study a high-temperature electroweak baryogenesis model using avoided deconfinement in further detail.

\subsection{High scale baryogenesis}

As discussed in section 2, in the usual RS model the high temperature phase is described by an AdS black hole, before a phase transition to the RS phase at a temperature around the $\mathrm{TeV}$ scale or below. The period of supercooling accompanying the phase transition significantly dilutes any pre-existing baryon asymmetry [33]. This has motivated consideration of baryogenesis mechanisms that combine the electroweak and RS phase transitions $[29,30,82]$. Baryogenesis mechanisms which operate at temperatures significantly above the $\mathrm{TeV}$ scale are difficult to realise in the RS model. This is not the case, however, for the $\mathrm{AD}$ model, as the universe is never in the $\mathrm{BH}$ phase after inflation and does not undergo a period of supercooling.

At high temperature the radion is stabilised closer to the UV brane. This means that fields localised toward the IR brane may have significant overlap with UV- localised fields at early times, with the UV and IR sectors then decoupling at low temperature. This allows for the possibility of having baryogenesis occur due to interactions between the IR 


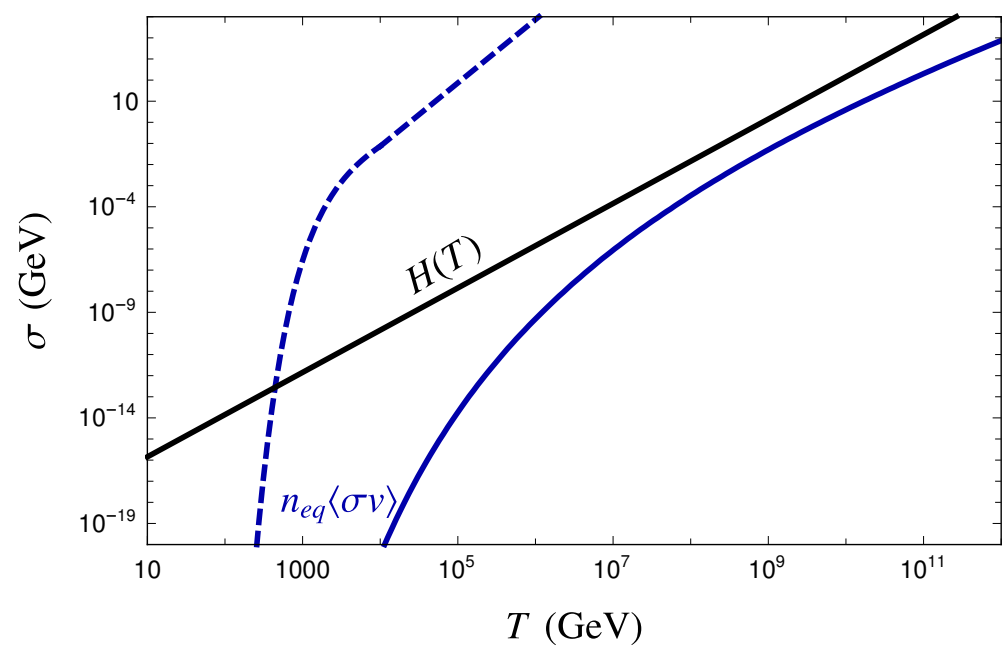

Figure 4. Dark matter annihilation rate for $m_{\chi}(T=0)=10 \mathrm{TeV}$, and $\alpha_{\mathrm{DM}} \sim 10^{-2}$ in the AD model (solid blue line) and without radion dependence (dashed blue line). The AD model parameters are as in equation (5.3). The Hubble rate assuming radiation domination and $g_{*}=106.75$ is shown in black.

and UV fields, which have $\mathcal{O}(1)$ couplings in the early universe but whose interactions are negligibly small after the radion has settled to its zero-temperature expectation value.

\subsection{WIMP freeze-in}

The relic abundance of a particle with weak scale mass and interactions in standard cosmology turns out to be a good estimate for the observed dark matter abundance. This has led to the WIMP paradigm, which is supported by the idea that new physics at the weak scale is motivated by the electroweak hierarchy problem. In this light, we expect a WIMP in the RS model to be associated with a field localised to the IR brane. Thus, avoided deconfinement may have significant implications for the WIMP freeze-out in such cases in fact, we show that particles with weak scale interactions can have a freeze-in mechanism.

As noted above, the scales on the IR brane are proportional to $\mu(T)$ and if the dark matter particle $\chi$ lives on the IR brane, the quantity $m_{\chi}(T) / T$ changes by only an $\mathrm{O}(1)$ amount during the phase of avoided deconfinement. In particular, the thermal abundance of $\chi$ can be Boltzmann suppressed for the entire cosmic history.

If the annihilation rate of dark matter is set by a weak coupling $\alpha_{\mathrm{DM}}$, then the equilibrium annihilation rate in early cosmology can be estimated as (assuming $m_{\chi}(0)>T_{c}$ ),

$n_{\mathrm{eq}}\langle\sigma v\rangle \sim \frac{\pi \alpha_{\mathrm{DM}}^{2}}{m_{\chi}(T)^{2}}\left(m_{\chi}(T) T\right)^{3 / 2} \exp \left[-\frac{m_{\chi}(T)}{T}\right] \sim T \frac{\pi \alpha_{\mathrm{DM}}^{2}}{m_{\chi}(0)^{2}} \exp \left[-\frac{m_{\chi}(0)}{c T_{c}}\right]+O\left(\epsilon \log \left(T / T_{c}\right)\right)$,

where we have ignored $\mathcal{O}(1)$ factors to highlight the scaling behavior. The form of the annihilation rate being nearly proportional to $T$ follows from the approximate conformal symmetry. Figure 4 shows the different annihilation rates as a function of temperature in the AD model and without radion dependence. 
The annihilation rate decreases slower than the Hubble rate, which decreases as $T^{2}$ in radiation domination, as shown in figure 4 . Even though the zero-temperature annihilation rate is weak-scale, the high-temperature annihilation rate can be out of equilibrium because it is Boltzmann suppressed. After the IR brane is stabilized at $T \sim T_{c}, m_{\chi}(T) \sim m_{\chi}(0)$, and the equilibrium annihilation rate drops exponentially with temperature. If the Hubble rate around $T=T_{c}$ is larger than the equilibrium annihilation rate, this implies that:

$$
\frac{m_{\chi}(0)}{T_{c}} \gtrsim \log \frac{M_{\mathrm{pl}}}{T_{c}}
$$

and the annihilation is never in thermal equilibrium, so the abundance is set by freeze in. Note that the DM-SM coupling can be sizeable, so that we can detect $\chi$ in direct and indirect detection experiments as a WIMP. However the usual relic abundance calculation would not apply. This can potentially open up a large parameter space for simple WIMPs like the Wino-like electroweak triplet, or other heavy WIMP candidates which would have a large abundance in the standard freeze out history.

\subsection{QCD phase transition and the QCD axion}

The QCD phase transition in the AD model may also be modified from the usual picture if $T_{c}$ is below the QCD scale. In order to achieve this in our model while satisfying the bounds on the radion and $s$ masses requires a large number of AD scalars. However, as was the case for the electroweak phase transition, even for $T_{c}$ slightly below the QCD scale the QCD phase transition may occur at temperatures far above the $\mathrm{TeV}$ scale. This may be able to reproduce some of the non-standard QCD dynamics discussed in refs. [83-85].

A cosmology where QCD confinement occurs at high temperatures, when $\Lambda_{\mathrm{QCD}}(T) \gg$ $\Lambda_{\mathrm{QCD}}(0)$, can also have dramatic consequences for the abundance of the QCD axion. The axion field can have various $5 \mathrm{D}$ origins; one simple possibility is that it is the fifth component of $\mathrm{U}(1)$ gauge field in 5D. Irrespective of its origin, the large decay constant of the axion suggests that its wavefunction is localised near the UV brane. Therefore, we can safely assume that $f_{a}$ is largely temperature independent. In the confined phase of QCD, but with a temperature-dependent confinement scale, the axion mass is given by,

$$
m_{a}(T)=\frac{f_{\pi}(T) m_{\pi}(T)}{f_{a}}
$$

The axion starts oscillating around the epoch of QCD confinement at a temperature $T_{\mathrm{osc}}$ which is defined by $m_{a}\left(T_{\mathrm{osc}}\right) \simeq H\left(T_{\mathrm{osc}}\right)$. The axion abundance at the onset of oscillation is

$$
\rho_{a}\left(T_{\mathrm{osc}}\right) \sim m_{a}^{2}\left(T_{\mathrm{osc}}\right) f_{a}^{2} \theta_{i}^{2}
$$

where $\theta_{i}$ is the initial misalignment angle. The mass of the axion continues to decrease due to the temperature dependence of $\Lambda_{\mathrm{QCD}}(T) \sim \mu(T)$. In the adiabatic approximation $\dot{m} \ll m^{2}$, the number density of the axion scales as $a^{-3}$, and the mass redshifts as $\sim a^{-2}$, so the axion energy density redshifts approximately as $a^{-5}$ in this epoch, whereas the background energy density is redshifting as $a^{-4}$. This can reduce the axion abundance dramatically. 


\subsection{Gravitational waves}

The absence of a first order confinement phase transition is a necessary feature of this mechanism that distinguishes it from the standard RS model. The RS phase transition results in a gravitational wave signal which will be absent in the avoided deconfinement model $[23,31]$. The RS phase transition also leads to a drop in $g_{*}$ of order $N^{2}$ as a result of degrees of freedom confining and freezing out. If there is an observable background of gravitational waves, such as from a cosmic string network [86, 87] or inflation [88-90], this change in $g_{*}$ is observable as a relative decrease in the power in modes which were below the horizon scale prior to the phase transition. The absence of these gravitational wave signals could be used to distinguish the AD model from RS models which do undergo a phase transition. Furthermore, the addition of the AD scalars, which are necessarily light degrees of freedom due to the bound (4.1), also leads to a potentially observable change in $g_{*}$ in the early universe for $N_{s}$ as low as $N_{s} \sim 10$ and masses around the GeV scale.

In addition to modifying the RS phase transition, in our set up there are additional phase transitions associated with the $s$ fields, which can be first order and can each happen at slightly different temperatures. This can give us interesting forest of GW signals with a spectrum that is different from the one expected from a single phase transition. As noted above, the electroweak and/or the QCD phase transition may also be made first order and can happen at very high temperatures, predicting a gravitational wave signature from these phase transitions as well.

\section{Discussion}

In this work we have described a mechanism which addresses the cosmological problem of eternal inflation due to suppressed confinement transitions in the RS model. The standard RS model is described at high temperature by an AdS black hole, with a transition to the RS phase proceeding via a first order phase transition which is exponentially suppressed by the large number $N^{2}$. We have shown that this situation can be avoided by introducing new scalars localised in the IR which generate a potential that stabilises the IR brane at high temperatures. Provided the universe exits inflation in the RS phase, it remains there, never entering the $\mathrm{BH}$ phase.

There are a number of issues that would be worth exploring further. It would be interesting to understand this phenomenon in a $4 \mathrm{D}$ field theory example. The additional scalars that we have introduced on the IR brane are expected to be emergent degrees of freedom in the $4 \mathrm{D}$ theory that appear after confinement. In such an example the thermal effect of these scalars will be to drive the confinement scale itself to higher values. This may provide a new insights on the problem of confinement.

There are also various phenomenological applications of this mechanism which we have merely touched upon in this paper. Avoided Deconfinement changes the cosmological history in a unique way, where from the IR brane point of view it is a model with effectively a low reheat temperature, but from the UV brane point of view the temperature can get arbitrarily high. This allows us to build realizations where the electroweak and/or QCD phase transition happen at very high temperatures. Since the cosmology at high 
temperatures is modified, we have shown that the mechanism for generating the abundance of various species such as WIMP dark matter, axion dark matter or baryogenesis can be significantly modified. An interesting future direction would be to build explicit models which realize these mechanisms, and study their phenomenological signatures.

Gravitational waves are a powerful experimental tool for studying very early universe cosmology, both in terms of new sources of the waves as well as modifications of propagation of gravitational waves in the early universe plasma. Modifications of the Randall-Sundrum phase transition, or the electroweak/QCD phase transition can change the expectations of GW signals from these phase transitions; these phase transitions are also associated with a change of number of degrees of freedom in the plasma, which may also be detectable in the GW spectrum. Even if a large- $N$ confining gauge group is part of a dark sector decoupled from the standard model, these gravitational wave signatures can provide important information about these sectors. Thus, the detailed phenomenological predictions of avoided deconfinement would be important to study further even in this more general situation.

\section{Acknowledgments}

We would like to thank Raman Sundrum and Soubhik Kumar for useful discussions and comments on the manuscript. We are grateful to Anson Hook, Lisa Randall, Matt Reece and John March-Russell for useful conversations. PA is supported by the STFC under Grant No. ST/T000864/1. MN is funded by a joint Clarendon and Sloane-Robinson scholarship from Oxford University and Keble college.

\section{A Bounce action for deconfining phase transition}

In this appendix we estimate the bounce action, $B$, which determines the transition rate from the $\mathrm{AD}$ phase in the high-temperature regime to the AdS-S or deconfined phase. At high temperatures the phase transition from the AD phase to the black hole phase proceeds at a rate

$$
\Gamma \simeq T^{4} e^{-B}
$$

If this is larger than $H^{4}$, where $H$ is the hubble rate, then this indicates that the AD phase is unstable. This defines a maximum temperature $T_{\max }$ above which the $\mathrm{AD}$ mechanism no longer works, but we will find that tunnelling only becomes significant at temperatures equal to the temperature which defines the classical instability of the model (defined in equation (3.20)), up to $\mathcal{O}(1 / N)$ corrections.

In order to determine $B$ we make the approximation that the action is dominated by the dynamics of the radion and neglect the contribution from the gravitational portion of the action. The justification for this is that the gravitational action scales as $N^{2}$ with no further enhancement from small or large parameters, while the contribution to the bounce from the radion, as we show below, scales like $N^{2} \lambda^{-1 / 2}$ for a weak coupling $\lambda$. In this 


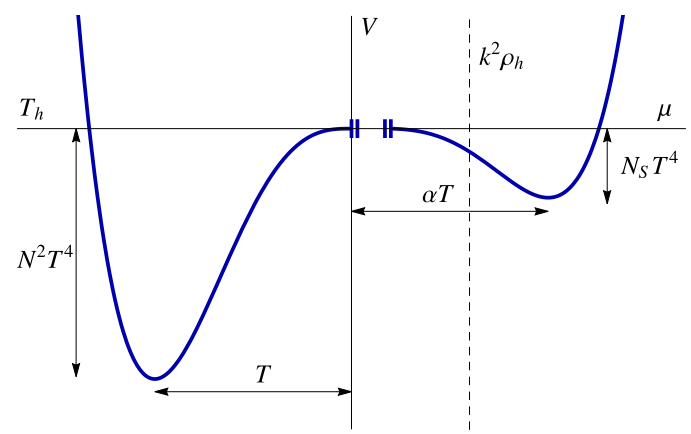

Figure 5. Plot showing the parametric dependence of the potential in the AD model. The right hand side shows the radion potential at high temperature, with depth set by $N_{s} T^{4}$ and width set by $\langle\mu\rangle=\alpha(T) T$ and the left hand side is the potential for the black hole hawking temperature $T_{h}$, with width set by $T$ and depth of order $N^{2} T^{4}$. The dashed line shows the would-be horizon position on the radion side of the potential and the break in the curve indicates the region where EFT control is lost.

approximation the relevant Euclidean action is

$$
\begin{aligned}
S_{E} & =\frac{N^{2}}{4 \pi} \int_{0}^{T^{-1}} d t_{E} \int r^{2} d r\left[(\partial \mu)^{2}-\lambda_{1} T^{2} \mu^{2}+\lambda_{2} \mu^{4}\right] \\
\lambda_{1} & =\frac{2 \pi^{2} N_{s} \lambda_{s} v_{s}^{2}}{3 N^{2}} \\
\lambda_{2} & =\frac{64 \pi^{2}\left(v_{\mathrm{ir}}-v_{\mathrm{uv}}\right)^{2}}{N^{2}}
\end{aligned}
$$

where we have explicitly scaled out the factor of $N^{2}$. At high temperature the minimum of the radion potential is well-approximated by equation (3.17)

$$
\mu \simeq \alpha(T) T, \quad \alpha(T)=\frac{\mu_{\mathrm{TeV}}}{\left(c T_{c} T^{\epsilon}\right)^{\frac{1}{1+\epsilon}}} .
$$

After rescaling the co-ordinates and radion field as $\mu=\alpha T \tilde{\mu}, x_{E}=T^{-1} \tilde{x}_{E}$ the action can be written as:

$$
S_{E}=\frac{\alpha^{2} N^{2}}{4 \pi} \int_{0}^{1} d \tilde{t}_{E} \int \tilde{r}^{2} d \tilde{r}\left[(\tilde{\partial} \tilde{\mu})^{2}-\lambda_{1} \tilde{\mu}^{2}+\lambda_{2} \alpha^{2} \tilde{\mu}^{4}\right] .
$$

The equation of motion for $\tilde{\mu}$ then implies that $\tilde{\mu}$ varies by an $\mathcal{O}(1)$ amount over a distance of order $\Delta \tilde{r} \sim \lambda_{1}^{-1 / 2} \gg 1$, i.e.

$$
\left|\frac{\partial \tilde{\mu}}{\partial \tilde{r}}\right| \sim \lambda_{1}^{1 / 2}
$$

We then make the conservative estimate that the bounce solution only requires the radion to vary by an amount given by

$$
\Delta \mu=\alpha(T) T-k^{2} \rho_{h},
$$

which amounts to moving the IR brane from its stabilised location to the position of the would-be horizon. In figure 5 this corresponds to the radion varying from its value at the 
minimum of the potential to the dashed line, as opposed to a bounce analogous to the one proposed in [20] which involves the radion varying to $\mu=0$ over the bounce trajectory. In terms of $\tilde{\mu}$ this is

$$
\delta \tilde{\mu}=1-\frac{\pi}{\alpha},
$$

which approaches 0 logarithmically (indicating that the IR brane is becoming classically unstable) as $T$ approaches $T_{\max }$.

With this estimate the characteristic radius of the tunnelling configuration will be $\tilde{R}_{b} \sim(\delta \tilde{\mu}) \lambda_{1}^{-1 / 2}$. For $\tilde{R}_{b} \gg 1$ we expect the bounce solution to be the $O(3)$ symmetric $\left(\tilde{t}_{E}\right.$-independent) configuration, while for $\tilde{R}_{b} \ll 1$ the solution will obey an $O(4)$ symmetry and depend on the variable $\tilde{\rho}=\sqrt{\tilde{t}_{E}^{2}+\tilde{r}^{2}}$. The $O(4)$ bounce solution therefore only becomes dominant for $\delta \tilde{\mu} \ll \lambda^{1 / 2} \sim 1 / N$ at which point the IR brane is close to becoming classically unstable anyway, so only the $O(3)$ symmetric bounce is relevant for computing the lifetime of the AD phase. The integrals determining the bounce action scale as

$$
\begin{aligned}
\int \tilde{r}^{2} d \tilde{r}\left((\tilde{\partial} \tilde{\mu})^{2}-\lambda_{1} \tilde{\mu}^{2}\right) & =\lambda_{1}^{-1 / 2} c_{1} \\
\int \tilde{r}^{2} d \tilde{r}\left(\lambda_{2} \alpha^{2} \tilde{\mu}^{4}\right) & =\lambda_{1}^{-3 / 2} \lambda_{2} c_{2} .
\end{aligned}
$$

where $c_{1}, c_{2}$ are $\mathcal{O}(1)$ coefficients. We can then estimate the bounce to be:

$$
\begin{aligned}
B & \simeq \frac{\alpha^{2} N^{2}(\delta \tilde{\mu})^{3} \lambda_{1}^{-3 / 2}}{4 \pi}\left[c_{1} \lambda_{1}+c_{2} \lambda_{2} \alpha^{2}\right] \\
& \simeq \frac{\sqrt{3} \alpha^{2} N^{3}(\delta \tilde{\mu})^{3}}{4 \sqrt{2} \pi^{2}\left(N_{s} \lambda_{s} v_{s}^{2}\right)^{1 / 2}}\left[c_{1}+c_{2} \frac{96 \alpha^{2}\left(v_{\mathrm{ir}}-v_{\mathrm{uv}}\right)^{2}}{N_{s} \lambda_{s} v_{s}^{2}}\right] .
\end{aligned}
$$

The bounce action is therefore suppressed by the factor $N^{3}$ and additionally by inverse powers of $v_{s}$. The lifetime of the metastable $\mathrm{AD}$ vacuum is much larger than Hubble for temperatures up until $\delta \tilde{\mu} \lesssim \mathcal{O}(1 / N)$, meaning we can safely ignore the tunneling rate from the $\mathrm{AD}$ to deconfined phase and consider only the classical instability of the model.

Open Access. This article is distributed under the terms of the Creative Commons Attribution License (CC-BY 4.0), which permits any use, distribution and reproduction in any medium, provided the original author(s) and source are credited.

\section{References}

[1] G. 't Hooft, A Planar Diagram Theory for Strong Interactions, Nucl. Phys. B 72 (1974) 461 [INSPIRE].

[2] J.M. Maldacena, The Large $N$ limit of superconformal field theories and supergravity, Adv. Theor. Math. Phys. 2 (1998) 231 [Int. J. Theor. Phys. 38 (1999) 1113] [hep-th/9711200] [INSPIRE].

[3] E. Witten, Anti-de Sitter space and holography, Adv. Theor. Math. Phys. 2 (1998) 253 [hep-th/9802150] [INSPIRE]. 
[4] S.S. Gubser, I.R. Klebanov and A.M. Polyakov, Gauge theory correlators from noncritical string theory, Phys. Lett. B 428 (1998) 105 [hep-th/9802109] [InSPIRE].

[5] L. Randall and R. Sundrum, A Large mass hierarchy from a small extra dimension, Phys. Rev. Lett. 83 (1999) 3370 [hep-ph/9905221] [INSPIRE].

[6] W.D. Goldberger and M.B. Wise, Modulus stabilization with bulk fields, Phys. Rev. Lett. 83 (1999) 4922 [hep-ph/9907447] [INSPIRE].

[7] M.A. Luty and R. Sundrum, Hierarchy stabilization in warped supersymmetry, Phys. Rev. D 64 (2001) 065012 [hep-th/0012158] [INSPIRE].

[8] H.L. Verlinde, Holography and compactification, Nucl. Phys. B 580 (2000) 264 [hep-th/9906182] [INSPIRE].

[9] C.S. Chan, P.L. Paul and H.L. Verlinde, A Note on warped string compactification, Nucl. Phys. B 581 (2000) 156 [hep-th/0003236] [InSPIRE].

[10] F. Brümmer, A. Hebecker and E. Trincherini, The Throat as a Randall-Sundrum model with Goldberger-Wise stabilization, Nucl. Phys. B 738 (2006) 283 [hep-th/0510113] [INSPIRE].

[11] L. Randall, The Boundaries of KKLT, Fortsch. Phys. 68 (2020) 1900105 [arXiv: 1912.06693] [INSPIRE].

[12] S. Kachru, R. Kallosh, A.D. Linde and S.P. Trivedi, de Sitter vacua in string theory, Phys. Rev. D 68 (2003) 046005 [hep-th/0301240] [INSPIRE].

[13] I.R. Klebanov and E. Witten, Superconformal field theory on three-branes at a Calabi-Yau singularity, Nucl. Phys. B 536 (1998) 199 [hep-th/9807080] [INSPIRE].

[14] I.R. Klebanov and A.A. Tseytlin, Gravity duals of supersymmetric $\mathrm{SU}(N) \times \mathrm{SU}(N+M)$ gauge theories, Nucl. Phys. B 578 (2000) 123 [hep-th/0002159] [INSPIRE].

[15] I.R. Klebanov and M.J. Strassler, Supergravity and a confining gauge theory: Duality cascades and chi SB resolution of naked singularities, JHEP 08 (2000) 052 [hep-th/0007191] [INSPIRE].

[16] I. Bena, E. Dudas, M. Graña and S. Lüst, Uplifting Runaways, Fortsch. Phys. 67 (2019) 1800100 [arXiv: 1809.06861] [INSPIRE].

[17] S. Kachru, M. Kim, L. Mcallister and M. Zimet, de Sitter Vacua from Ten Dimensions, arXiv: 1908.04788 [INSPIRE].

[18] S.W. Hawking and D.N. Page, Thermodynamics of Black Holes in anti-de Sitter Space, Commun. Math. Phys. 87 (1983) 577 [INSPIRE].

[19] E. Witten, Anti-de Sitter space, thermal phase transition, and confinement in gauge theories, Adv. Theor. Math. Phys. 2 (1998) 505 [hep-th/9803131] [INSPIRE].

[20] P. Creminelli, A. Nicolis and R. Rattazzi, Holography and the electroweak phase transition, JHEP 03 (2002) 051 [hep-th/0107141] [INSPIRE].

[21] J. Kaplan, P.C. Schuster and N. Toro, Avoiding an Empty Universe in RS I Models and Large- $N$ Gauge Theories, hep-ph/0609012 [INSPIRE].

[22] S.S. Gubser, AdS/CFT and gravity, Phys. Rev. D 63 (2001) 084017 [hep-th/9912001] [INSPIRE].

[23] L. Randall and G. Servant, Gravitational waves from warped spacetime, JHEP 05 (2007) 054 [hep-ph/0607158] [INSPIRE]. 
[24] G. Nardini, M. Quirós and A. Wulzer, A Confining Strong First-Order Electroweak Phase Transition, JHEP 09 (2007) 077 [arXiv:0706.3388] [INSPIRE].

[25] T. Konstandin, G. Nardini and M. Quirós, Gravitational Backreaction Effects on the Holographic Phase Transition, Phys. Rev. D 82 (2010) 083513 [arXiv:1007.1468] [INSPIRE].

[26] T. Konstandin and G. Servant, Cosmological Consequences of Nearly Conformal Dynamics at the TeV scale, JCAP 12 (2011) 009 [arXiv:1104.4791] [INSPIRE].

[27] B.M. Dillon, B.K. El-Menoufi, S.J. Huber and J.P. Manuel, Rapid holographic phase transition with brane-localized curvature, Phys. Rev. D 98 (2018) 086005 [arXiv: 1708.02953] [INSPIRE].

[28] B. von Harling and G. Servant, QCD-induced Electroweak Phase Transition, JHEP 01 (2018) 159 [arXiv: 1711.11554] [INSPIRE].

[29] S. Bruggisser, B. Von Harling, O. Matsedonskyi and G. Servant, Baryon Asymmetry from a Composite Higgs Boson, Phys. Rev. Lett. 121 (2018) 131801 [arXiv: 1803. 08546] [INSPIRE].

[30] S. Bruggisser, B. Von Harling, O. Matsedonskyi and G. Servant, Electroweak Phase Transition and Baryogenesis in Composite Higgs Models, JHEP 12 (2018) 099 [arXiv: 1804.07314] [INSPIRE].

[31] E. Megías, G. Nardini and M. Quirós, Cosmological Phase Transitions in Warped Space: Gravitational Waves and Collider Signatures, JHEP 09 (2018) 095 [arXiv:1806. 04877] [INSPIRE].

[32] D. Bunk, J. Hubisz and B. Jain, A Perturbative RS I Cosmological Phase Transition, Eur. Phys. J. C 78 (2018) 78 [arXiv:1705.00001] [InSPIRE].

[33] P. Baratella, A. Pomarol and F. Rompineve, The Supercooled Universe, JHEP 03 (2019) 100 [arXiv: 1812.06996] [INSPIRE].

[34] K. Agashe, P. Du, M. Ekhterachian, S. Kumar and R. Sundrum, Cosmological Phase Transition of Spontaneous Confinement, JHEP 05 (2020) 086 [arXiv:1910.06238] [INSPIRE].

[35] K. Fujikura, Y. Nakai and M. Yamada, A more attractive scheme for radion stabilization and supercooled phase transition, JHEP 02 (2020) 111 [arXiv:1910.07546] [INSPIRE].

[36] A. Azatov and M. Vanvlasselaer, Phase transitions in perturbative walking dynamics, JHEP 09 (2020) 085 [arXiv: 2003.10265] [INSPIRE].

[37] E. Megía, G. Nardini and M. Quirós, Gravitational Imprints from Heavy Kaluza-Klein Resonances, Phys. Rev. D 102 (2020) 055004 [arXiv:2005.04127] [InSPIRE].

[38] K. Agashe, P. Du, M. Ekhterachian, S. Kumar and R. Sundrum, Phase Transitions from the Fifth Dimension, JHEP 02 (2021) 051 [arXiv: 2010.04083] [INSPIRE].

[39] B. Hassanain, J. March-Russell and M. Schvellinger, Warped Deformed Throats have Faster (Electroweak) Phase Transitions, JHEP 10 (2007) 089 [arXiv:0708.2060] [INSPIRE].

[40] S. Weinberg, Gauge and Global Symmetries at High Temperature, Phys. Rev. D 9 (1974) 3357 [INSPIRE].

[41] P. Meade and H. Ramani, Unrestored Electroweak Symmetry, Phys. Rev. Lett. 122 (2019) 041802 [arXiv: 1807.07578] [INSPIRE].

[42] I. Baldes and G. Servant, High scale electroweak phase transition: baryogenesis \& symmetry non-restoration, JHEP 10 (2018) 053 [arXiv:1807.08770] [INSPIRE]. 
[43] A. Glioti, R. Rattazzi and L. Vecchi, Electroweak Baryogenesis above the Electroweak Scale, JHEP 04 (2019) 027 [arXiv:1811.11740] [INSPIRE].

[44] O. Matsedonskyi and G. Servant, High-Temperature Electroweak Symmetry Non-Restoration from New Fermions and Implications for Baryogenesis, JHEP 09 (2020) 012 [arXiv: 2002.05174] [INSPIRE].

[45] P. Langacker and S.-Y. Pi, Magnetic Monopoles in Grand Unified Theories, Phys. Rev. Lett. 45 (1980) 1 [INSPIRE].

[46] P. Salomonson, B.S. Skagerstam and A. Stern, On the Primordial Monopole Problem in Grand Unified Theories, Phys. Lett. B 151 (1985) 243 [InSPIRE].

[47] G.R. Dvali, A. Melfo and G. Senjanović, Is There a monopole problem?, Phys. Rev. Lett. 75 (1995) 4559 [hep-ph/9507230] [INSPIRE].

[48] G.R. Dvali and G. Senjanović, Is there a domain wall problem?, Phys. Rev. Lett. 74 (1995) 5178 [hep-ph/9501387] [INSPIRE].

[49] R.N. Mohapatra and G. Senjanović, Soft CP-violation at High Temperature, Phys. Rev. Lett. 42 (1979) 1651 [INSPIRE].

[50] R.N. Mohapatra and G. Senjanović, Broken Symmetries at High Temperature, Phys. Rev. D 20 (1979) 3390 [INSPIRE].

[51] J. Orloff, The UV price for symmetry nonrestoration, Phys. Lett. B 403 (1997) 309 [hep-ph/9611398] [INSPIRE].

[52] N. Chai, S. Chaudhuri, C. Choi, Z. Komargodski, E. Rabinovici and M. Smolkin, Thermal Order in Conformal Theories, Phys. Rev. D 102 (2020) 065014 [arXiv:2005.03676] [INSPIRE].

[53] N. Arkani-Hamed, M. Porrati and L. Randall, Holography and phenomenology, JHEP 08 (2001) 017 [hep-th/0012148] [INSPIRE].

[54] R. Rattazzi and A. Zaffaroni, Comments on the holographic picture of the Randall-Sundrum model, JHEP 04 (2001) 021 [hep-th/0012248] [INSPIRE].

[55] C. Charmousis, R. Gregory and V.A. Rubakov, Wave function of the radion in a brane world, Phys. Rev. D 62 (2000) 067505 [hep-th/9912160] [InSPIRE].

[56] S.R. Coleman, The Fate of the False Vacuum. 1. Semiclassical Theory, Phys. Rev. D 15 (1977) 2929 [Erratum ibid. 16 (1977) 1248] [INSPIRE].

[57] A.D. Linde, Decay of the False Vacuum at Finite Temperature, Nucl. Phys. B 216 (1983) 421 [Erratum ibid. 223 (1983) 544] [INSPIRE].

[58] F. Coradeschi, P. Lodone, D. Pappadopulo, R. Rattazzi and L. Vitale, A naturally light dilaton, JHEP 11 (2013) 057 [arXiv: 1306.4601] [INSPIRE].

[59] W.D. Goldberger and M.B. Wise, Phenomenology of a stabilized modulus, Phys. Lett. B $\mathbf{4 7 5}$ (2000) 275 [hep-ph/9911457] [INSPIRE].

[60] A. Pomarol, O. Pujolàs and L. Salas, Holographic conformal transition and light scalars, JHEP 10 (2019) 202 [arXiv:1905.02653] [INSPIRE].

[61] F. Bigazzi, A. Caddeo, A.L. Cotrone and A. Paredes, Fate of false vacua in holographic first-order phase transitions, JHEP 12 (2020) 200 [arXiv:2008.02579] [INSPIRE]. 
[62] A.H. Guth and E.J. Weinberg, Could the Universe Have Recovered from a Slow First Order Phase Transition?, Nucl. Phys. B 212 (1983) 321 [InSPIRE].

[63] S. Fichet, Braneworld effective field theories - holography, consistency and conformal effects, JHEP 04 (2020) 016 [arXiv: 1912.12316] [INSPIRE].

[64] C. Delaunay, C. Grojean and J.D. Wells, Dynamics of Non-renormalizable Electroweak Symmetry Breaking, JHEP 04 (2008) 029 [arXiv:0711.2511] [INSPIRE].

[65] D. Curtin, P. Meade and H. Ramani, Thermal Resummation and Phase Transitions, Eur. Phys. J. C 78 (2018) 787 [arXiv: 1612.00466] [InSPIRE].

[66] Z. Chacko, R.K. Mishra, D. Stolarski and C.B. Verhaaren, Interactions of a Stabilized Radion and Duality, Phys. Rev. D 92 (2015) 056004 [arXiv: 1411.3758] [INSPIRE].

[67] T. Gherghetta and A. Pomarol, Bulk fields and supersymmetry in a slice of AdS, Nucl. Phys. $B 586$ (2000) 141 [hep-ph/0003129] [INSPIRE].

[68] R. Sundrum, Gravity's scalar cousin, hep-th/0312212 [InSPIRE].

[69] A. Hebecker and J. March-Russell, Randall-Sundrum II cosmology, AdS / CFT, and the bulk black hole, Nucl. Phys. B 608 (2001) 375 [hep-ph/0103214] [InSPIRE].

[70] K. Agashe, A. Delgado, M.J. May and R. Sundrum, RS1, custodial isospin and precision tests, JHEP 08 (2003) 050 [hep-ph/0308036] [INSPIRE].

[71] S. Casagrande, F. Goertz, U. Haisch, M. Neubert and T. Pfoh, Flavor Physics in the Randall-Sundrum Model: I. Theoretical Setup and Electroweak Precision Tests, JHEP 10 (2008) 094 [arXiv: 0807.4937] [INSPIRE].

[72] K. Agashe, G. Perez and A. Soni, Flavor structure of warped extra dimension models, Phys. Rev. D 71 (2005) 016002 [hep-ph/0408134] [INSPIRE].

[73] M. Bauer, S. Casagrande, U. Haisch and M. Neubert, Flavor Physics in the Randall-Sundrum Model: II. Tree-Level Weak-Interaction Processes, JHEP 09 (2010) 017 [arXiv: 0912.1625] [INSPIRE].

[74] K. Blum, M. Cliche, C. Csáki and S.J. Lee, WIMP Dark Matter through the Dilaton Portal, JHEP 03 (2015) 099 [arXiv: 1410.1873] [INSPIRE].

[75] CMS collaboration, Search for physics beyond the standard model in high-mass diphoton events from proton-proton collisions at $\sqrt{s}=13 \mathrm{TeV}$, Phys. Rev. D 98 (2018) 092001 [arXiv: 1809.00327] [INSPIRE].

[76] CMS collaboration, Combination of searches for Higgs boson pair production in proton-proton collisions at $\sqrt{s}=13 \mathrm{TeV}$, Phys. Rev. Lett. 122 (2019) 121803 [arXiv: 1811.09689] [INSPIRE].

[77] G.F. Giudice, Y. Kats, M. McCullough, R. Torre and A. Urbano, Clockwork/linear dilaton: structure and phenomenology, JHEP 06 (2018) 009 [arXiv:1711.08437] [INSPIRE].

[78] F. Abu-Ajamieh, J.S. Lee and J. Terning, The Light Radion Window, JHEP 10 (2018) 050 [arXiv: 1711.02697] [INSPIRE].

[79] E. Masso and R. Toldra, On a light spinless particle coupled to photons, Phys. Rev. D 52 (1995) 1755 [hep-ph/9503293] [INSPIRE].

[80] J. Jaeckel and M. Spannowsky, Probing MeV to $90 \mathrm{GeV}$ axion-like particles with LEP and LHC, Phys. Lett. B 753 (2016) 482 [arXiv:1509.00476] [inSPIRE]. 
[81] B. Döbrich, J. Jaeckel, F. Kahlhoefer, A. Ringwald and K. Schmidt-Hoberg, ALPtraum: ALP production in proton beam dump experiments, JHEP 02 (2016) 018 [arXiv: 1512.03069] [INSPIRE].

[82] T. Konstandin and G. Servant, Natural Cold Baryogenesis from Strongly Interacting Electroweak Symmetry Breaking, JCAP 07 (2011) 024 [arXiv:1104.4793] [INSPIRE].

[83] S. Ipek and T.M.P. Tait, Early Cosmological Period of QCD Confinement, Phys. Rev. Lett. 122 (2019) 112001 [arXiv: 1811.00559] [INSPIRE].

[84] D. Croon, J.N. Howard, S. Ipek and T.M.P. Tait, QCD baryogenesis, Phys. Rev. D 101 (2020) 055042 [arXiv:1911.01432] [INSPIRE].

[85] D. Berger, S. Ipek, T.M.P. Tait and M. Waterbury, Dark Matter Freeze Out during an Early Cosmological Period of QCD Confinement, JHEP 07 (2020) 192 [arXiv:2004.06727] [INSPIRE].

[86] Y. Cui, M. Lewicki, D.E. Morrissey and J.D. Wells, Cosmic Archaeology with Gravitational Waves from Cosmic Strings, Phys. Rev. D 97 (2018) 123505 [arXiv:1711.03104] [InSPIRE].

[87] Y. Cui, M. Lewicki, D.E. Morrissey and J.D. Wells, Probing the pre-BBN universe with gravitational waves from cosmic strings, JHEP 01 (2019) 081 [arXiv: 1808. 08968] [INSPIRE].

[88] Y. Watanabe and E. Komatsu, Improved Calculation of the Primordial Gravitational Wave Spectrum in the Standard Model, Phys. Rev. D 73 (2006) 123515 [astro-ph/0604176] [INSPIRE].

[89] R. Jinno, T. Moroi and K. Nakayama, Probing dark radiation with inflationary gravitational waves, Phys. Rev. D 86 (2012) 123502 [arXiv:1208.0184] [INSPIRE].

[90] K. Saikawa and S. Shirai, Primordial gravitational waves, precisely: The role of thermodynamics in the Standard Model, JCAP 05 (2018) 035 [arXiv: 1803.01038] [INSPIRE]. 